\title{
Stellar evolution with rotation. VII.
}

\section{Low metallicity models and the blue to red supergiant ratio in the SMC}

\author{
A. Maeder and G. Meynet
}

\begin{abstract}
Geneva Observatory 1290 Sauverny, Switzerland
e-mail: Georges. Meynet@obs . unige.ch
\end{abstract}

Received 15 March 2001 / Accepted 12 April 2001

\begin{abstract}
We calculate a grid of models with and without the effects of axial rotation for massive stars in the range of 9 to $60 M_{\odot}$ and metallicity $Z=0.004$ appropriate for the SMC. Remarkably, the ratios $\Omega / \Omega_{\text {crit }}$ of the angular velocity to the break-up angular velocity grow strongly during the evolution of high mass stars, contrary to the situation at $Z=0.020$. The reason is that at low $Z$, mass loss is smaller and the removal of angular momentum during evolution much weaker, also there is an efficient outward transport of angular momentum by meridional circulation. Thus, a much larger fraction of the stars at lower $Z$ reach break-up velocities and rotation may thus be a dominant effect at low $Z$. The models with rotation well account for the long standing problem of the large numbers of red supergiants observed in low $Z$ galaxies, while current models with mass loss were predicting no red supergiants. We discuss in detail the physical effects of rotation which favour a redwards evolution in the HR diagram. The models also predict large $\mathrm{N}$ enrichments during the evolution of high mass stars. The predicted relative $\mathrm{N}$-enrichments are larger at $Z$ lower than solar and this is in very good agreement with the observations for A-type supergiants in the SMC.
\end{abstract}

Key words. stars: evolution - stars: rotation - supergiant - magellanic clouds

\section{Introduction}

The aim of this work is to study the effects of rotation in massive stars at low metallicity $Z$ as in the SMC. There are 3 main problems emerging in this context. 1.- Firstly, we want to examine whether there are differences between the effects of rotation at low $Z$ and at solar metallicity. Surprisingly, we find some striking differences, which suggest that rotation may be a much more important effect in the evolution of stars at $Z$ lower than solar. 2.- Then, we want to address the problem of the number ratio $B / R$ of blue to red supergiants, which is one of the most severe problems in stellar evolution. As stated by Kippenhahn \& Weigert (1990) about stars on the blue loops in the $\mathrm{He}^{-}$ burning phase, the present phase " ... is a sort of magnifying glass, revealing relentlessly the faults of calculations of earlier phases". As long as the problem is not solved, we cannot correctly predict the populations of massive stars in galaxies at various metallicities $Z$, neither the spectral evolution of galaxies, nor the chemical yields. 3.- A third important question concerns the chemical abundances of massive stars at low metallicities. In particular, we recall that very large nitrogen enrichments have been found for

Send offprint requests to: A. Maeder, e-mail: Andre.Maeder@obs.unige.ch the A-type supergiants in the SMC (Venn 1998, 1999). There, the relative $N / H$ excesses can reach a factor of 10 , much larger than in the Galaxy. This is a strong constraint, which needs to be examined carefully.

Let us comment a bit more on the embarassing problem of the number ratio $\mathrm{B} / \mathrm{R}$, which has been reviewed by Langer \& Maeder (1995) and by Maeder \& Meynet (2000a). To make a long story short, the observations of nearby galaxies show that there are much more red supergiants at metallicities lower than solar, so that $\mathrm{B} / \mathrm{R}$ decreases steeply with decreasing metallicity (Humphreys \& McElroy 1984). Large differences in the $\mathrm{B} / \mathrm{R}$ ratio also exist between clusters in the Galaxy and in the SMC, where the red supergiants were found more numerous by an order of a magnitude (Meylan \& Maeder 1982).

There are no sets of models which correctly predict the observed trend of decreasing $\mathrm{B} / \mathrm{R}$ with decreasing $Z$, as emphasized by Langer \& Maeder (1995). All kind of models were examined: models with Schwarzschild's criterion (cf. Stothers \& Chin 1992), models with Schwarzschild's criterion and overshooting (cf. Schaller et al. 1992), models with Ledoux criterion (cf. Stothers \& Chin 1992; Brocato \& Castellani 1993), models with semiconvection (cf. Arnett 1991), models with semiconvective diffusion (cf. Langer \& Maeder 1995). The comparisons generally 
show that the models with Schwarzschild's criterion (with or without overshooting) may reproduce the observed B/R at solar metallicity, while they fail at lower $Z$. At the other extreme, the models with the Ledoux criterion and those with semiconvection reproduce well $\mathrm{B} / \mathrm{R}$ at the metallicities of the SMC, but they fail at higher $Z$. This shows that, at least in part, the problem is related to the size of the core and to the mixing efficiency outside the core.

Of course, other effects such as mass loss, convection, opacities and metallicities play a role. In particular, as evidenced by the Geneva grids of models (Meynet et al. 1994; cf. also Maeder 1981) a growth of mass loss favours the formation of more red supergiants in the He-burning phase. However, the $\mathrm{B} / \mathrm{R}$ problem in the $\mathrm{SMC}$ cannot be solved by mass loss, because the mass loss rates at lower $Z$, as in the SMC, are smaller than in the Galaxy and this produces fewer red supergiants.

In some recent works, it has been shown that the account of the axial rotation of stars changes all the model outputs; tracks in the HR diagram, lifetimes, surface abundances, etc. (Meynet \& Maeder 2000; Heger \& Langer 2000). Noticeably rotation, both by its effects on internal mixing and on mass loss was found to favour the redward motions. This is a positive indication and we now further explore it. A grid of stellar models with rotation in the range of 9 to $60 M_{\odot}$ and metallicity $Z=0.004$ was constructed. It will provide a basis for comparison with the SMC observations.

In Sect. 2, we briefly discuss the improvements brought in the model physics. In Sect. 3, we discuss the evolution of the internal rotation law $\Omega(r)$ and in Sect. 4 of the surface rotation velocities $v$. The models with zero rotation are briefly discussed in Sect. 5. In Sect. 6, the results for the $\mathrm{HR}$ diagram and the lifetimes are shown. Section 7 is devoted to the study of the $\mathrm{B} / \mathrm{R}$ ratio from the numerical models; we also account for the results in terms of the physical properties of stellar models. In Sect. 8, we analyze the chemical abundances at the stellar surface and compare the results to observations of A-type supergiants in the SMC. Section 9 gives the conclusions.

\section{Physics of the models}

We apply the treatment of the hydrostatic effects appropriate to differential rotation, as described by Meynet \& Maeder (1997). We recall that the classical treatment frequently applied is as a matter of fact not correct in the presence of shellular differential rotation. We also account for the rotational distortion and for the von Zeipel theorem. The $T_{\text {eff }}$ given here corresponds to the value as observed for an average orientation angle between the axis of rotation and the direction of the observer. We include the effects of shear diffusion (Zahn 1992; Maeder 1997) and the effects of the meridional circulation, as studied by Maeder \& Zahn (1998). The same physics of the models as described in Meynet \& Maeder (2000, Paper V) is used here, with a few improvements, which are mentioned below.

\subsection{Meridional circulation, shears and horizontal turbulence}

Meridional circulation plays a major role in the redistribution of the angular momentum in stars. Let us write the equations for the radial term of the vertical component $U(r)$ of the meridional circulation (cf. Maeder \& Zahn 1998), which will be essential for the following discussions,

$$
\begin{aligned}
& U(r)=\frac{P}{\overline{\rho g} C_{P} \bar{T}\left[\nabla_{\mathrm{ad}}-\nabla+(\varphi / \delta) \nabla_{\mu}\right]} \\
& \times\left\{\frac{L}{M_{\star}}\left(E_{\Omega}+E_{\mu}\right)+\frac{C_{P}}{\delta} \frac{\partial \Theta}{\partial t}\right\},
\end{aligned}
$$

with the current notations as given in the quoted paper. The main term in the braces in the second member is $E_{\Omega}$. If we ignore secondary terms, it behaves essentially like,

$$
E_{\Omega} \simeq \frac{8}{3}\left[1-\frac{\Omega^{2}}{2 \pi G \bar{\rho}}\right]\left(\frac{\Omega^{2} r^{3}}{G M}\right) .
$$

The overlined expressions like $\bar{\rho}$ mean the average over the considered equipotential. The term with the minus sign in the square bracket is the Gratton-Öpik term, which becomes important in the outer layers due to the decrease of the local density; it can produce negative values of $U(r)$. A negative $U(r)$ means a circulation going down along the polar axis and up in the equatorial plane, thus making an outwards transport of angular momentum. The positive or negative values of $U(r)$ play a major role in massive star evolution.

In Paper V we did not account for the effects of horizontal turbulence on the shears. This was done by Talon \& Zahn (1997). They found that the diffusion coefficient for the shears is modified by the horizontal turbulence. The change can be an increase or a decrease of the diffusion coefficient depending on the various parameters, as discussed below. Thus, we also have to include the developments by Talon \& Zahn (1997), we have

$$
\begin{aligned}
D= & \frac{\left(K+D_{\mathrm{h}}\right)}{\left[\frac{\varphi}{\delta} \nabla_{\mu}\left(1+\frac{K}{D_{\mathrm{h}}}\right)+\left(\nabla_{\mathrm{ad}}-\nabla_{\mathrm{rad}}\right)\right]} \\
& \times \frac{H_{\mathrm{p}}}{g \delta}\left[\alpha\left(0.8836 \Omega \frac{\mathrm{d} \ln \Omega}{\mathrm{d} \ln r}\right)^{2}-4\left(\nabla^{\prime}-\nabla\right)\right]
\end{aligned}
$$

with $\quad D_{\mathrm{h}} \simeq|r U(r)|$

where $D_{\mathrm{h}}$ is the coefficient of horizontal diffusion (cf. Zahn 1992). We ignore here the thermal coupling effects discussed by Maeder (1997) because they were found to be relatively small and they increase the numerical complexity. Interestingly, we see that in regions where $\nabla_{\mu} \simeq 0$, Eq. (3) leads us to replace $K$ by $\left(K+D_{\mathrm{h}}\right)$ in the usual expression (cf. Talon \& Zahn 1997), i.e. it reinforces slightly the diffusion in regions which are close to chemical homogeneity. On the contrary, in regions where $\nabla_{\mu}$ dominates with respect to $\left(\nabla_{\mathrm{ad}}-\nabla_{\mathrm{rad}}\right)$, the transport is proportional 
to $D_{\mathrm{h}}$ rather than to $K$, which is quite logical since the diffusion is then determined by $D_{\mathrm{h}}$ rather than by thermal effects. The above result shows the importance of the treatment for the meridional circulation, since in turn it determines the size of $D_{\mathrm{h}}$ and to some extent the diffusion by shears. The equation of transport of angular momentum and of chemical elements are followed explicitly.

\subsection{Mass loss rates and their dependence on rotation}

For the mass loss rates, the recent data by Kudritzki \& Puls (2000) for OB stars and B-type supergiants are applied, they are completed for A-F supergiants by the expressions by de Jager et al. (1988). For the red supergiants, there are a number of recent parametrizations proposed (cf. Willson 2000), from low to high rates. We choose finally the medium-high rates as they were proposed by Vanbeveren et al. (1998). For WR stars, the rates by Nugis \& Lamers (2000) were applied. These rates account for the clumping of matter in the winds from WR stars and they are lower by about a factor of 2 than those used in the Geneva grids (cf. Schaller et al. 1992).

We have to account for rotation effects on the mass loss rates. In our previous work (Meynet \& Maeder 2000), we used the expression derived by Langer (1998), based on the numerical models by Friend \& Abbott (1986). However, these models did not include the gravity darkening predicted by the von Zeipel formula. A new expression has been derived by the application of the wind theory over the surface of a rotating star, taking also into account various improvements in the model of a rotating star (Maeder \& Meynet 2000b). The ratio of the mass loss rate of a star rotating with an angular velocity $\Omega$ to that of a non rotating star at the same location in the HR diagram behaves as

$$
\frac{\dot{M}(\Omega)}{\dot{M}(0)}=\frac{(1-\Gamma)^{\frac{1}{\alpha}-1}}{\left[1-\frac{\Omega^{2}}{2 \pi G \rho_{\mathrm{m}}}-\Gamma\right]^{\frac{1}{\alpha}-1}} .
$$

The term $\Gamma$ is the usual Eddington factor for electron scattering opacities and $\rho_{\mathrm{m}}$ is the average density internal to the surface equipotential. The term $\frac{\Omega^{2}}{2 \pi G \rho_{\mathrm{m}}}$ is a function of the ratio of the rotational velocity to the break-up velocity

$\frac{\Omega^{2}}{2 \pi G \rho_{\mathrm{m}}} \simeq \frac{4}{9} \frac{v^{2}}{v_{\mathrm{crit}}^{2}}$,

with deviations of less than $\sim 3 \%$ up to $\frac{v}{v_{\text {crit }}}=0.8$. Here, $v_{\text {crit }}=\left(\frac{2}{3} \frac{G M}{R_{\mathrm{pb}}}\right)^{\frac{1}{2}}$ and $R_{\mathrm{pb}}$ is the polar radius at break-up $\left(R_{\mathrm{pb}}\right.$ can be taken equal to the radius of the non-rotating star). The coefficient $\alpha$ in Eq. (5) is a force multiplier, which depends on $T_{\text {eff }}$. We use the empirical force multipliers derived by Lamers et al. (1995): $\alpha=0.52,0.24,0.17$ and 0.15 , for $\log T_{\text {eff }} \geq 4.35,4.30,4.00$ and 3.90 respectively.

Of course, we have to account for the fact that the empirical values for the mass loss rates used for non-rotating stars are based on stars covering the whole range of rotational velocities. Thus, we must apply a reduction factor to the empirical rates to make them correspond to the non rotating case. After convolution of the effects described by Eq. (5) over the observed distribution of rotational velocities, taking also into account that the axes of orientation are randomly distributed, we estimate that the average correcting factor is equal to about 0.8 , which has to be applied to the mass loss rates for the Main-Sequence (MS) OB stars. For post-MS stars, the mass loss rates are too uncertain anyway, and the multiplying correction factor is almost unity, so that we do not apply any correction.

\subsection{Initial composition, opacities, nuclear reactions}

For a given metallicity $Z$ (in mass fraction), we estimate the initial helium mass fraction $Y$ from the relation $Y=Y_{\mathrm{p}} \cdot \Delta Y / \Delta Z Z$, where $Y_{\mathrm{p}}$ is the primordial helium abundance and $\Delta Y / \Delta Z$ the slope of the helium-to-metal enrichment law. Values of $Y_{\mathrm{p}}$ between 0.23 and 0.24 are given in the literature (see e.g. Pagel et al. 1992; Izotov et al. 1997; Peimbert et al. 2000). Observations of HII regions in blue compact dwarf galaxies indicate values for $\Delta Y / \Delta Z$ equal to $1.7 \pm 0.9$ (Izotov et al. 1997), $2.3 \pm 1.0$ (Thuan \& Izotov 1998). Such values are in agreement with the $\Delta Y / \Delta Z$ value of $2.3 \pm 1.5$ derived by Fernandes et al. (1998) from the study of nearby visual binary stars. Here we set $Y_{\mathrm{p}}=0.23$ and $\Delta Y / \Delta Z=2.25$ as in the recent grids of stellar models by Girardi et al. (2000). For the metallicity $Z=0.004$ considered in this work, we thus obtain $X=0.757$ and $Y=0.239$. For the mixture of the heavy elements, we adopted the same mixture as the one used to compute the opacity tables. Opacities are from Iglesias \& Rogers (1996) complemented at low temperatures with the molecular opacities of Alexander (http://web.physics.twsu.edu/alex/wwwdra.htm).

The rates for the charged particle reactions are taken from the new NACRE compilation (Angulo et al. 1999). For the reaction ${ }^{12} \mathrm{C}(\alpha, \gamma){ }^{16} \mathrm{O}$ and for the range of temperatures corresponding to the He-burning phase, the NACRE rate is a factor two higher than the rate of Caughlan \& Fowler (1988) and amounts to about $80 \%$ of the rate adopted in Paper $\mathrm{V}$ and taken from Caughlan et al. (1985). Finally, let us recall that as in Paper V, we adopted the Schwarzschild criterion for convection without overshooting.

Thus, with respect to Paper $\mathrm{V}$, in addition to the improvements of the physics describing the effects of rotation on the mixing of the chemical elements and on the mass loss rates, we have updated the mass loss rates (see previous section), the initial composition, the opacities and the nuclear reaction rates. In order to evaluate the importance of the changes which are not linked to the physics of rotation, we have computed a non-rotating $20 M_{\odot}$ model at solar metallicity with the physics included in this paper (see Fig. 8 below). We obtain that the present $20 M_{\odot}$ model at solar metallicity follows a very similar track in 


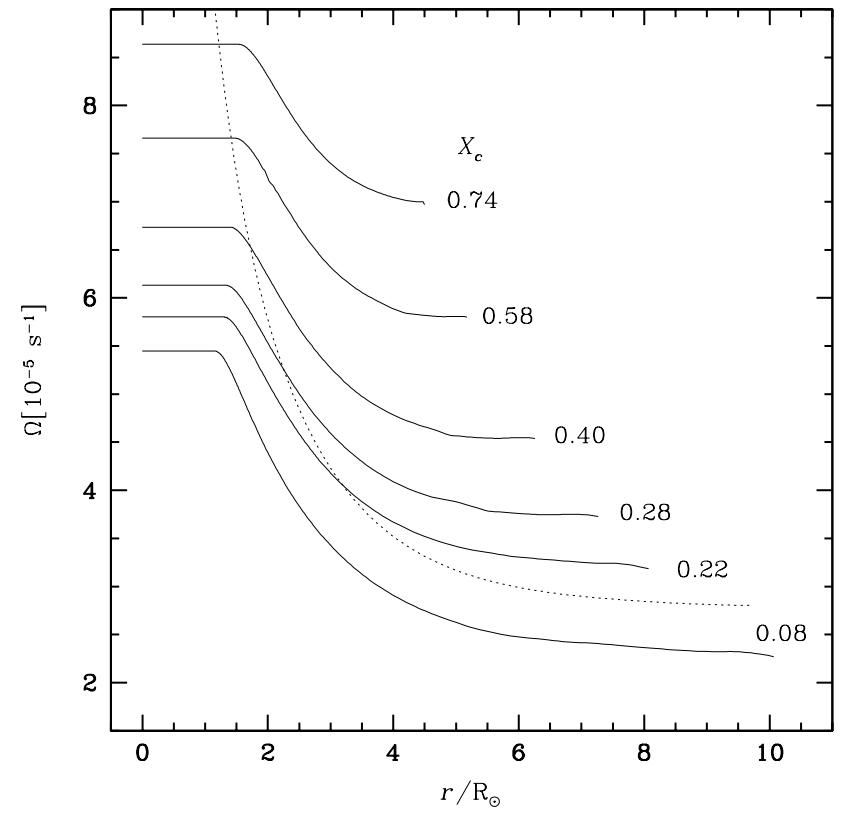

Fig. 1. Evolution of the angular velocity $\Omega$ as a function of the distance to the center in a $20 M_{\odot}$ star with $v_{\text {ini }}=300 \mathrm{~km} \mathrm{~s}^{-1}$ and $Z=0.004 . X_{\mathrm{c}}$ is the hydrogen mass fraction at the center. The dotted line shows the profile when the He-core contracts at the end of the $\mathrm{H}$-burning phase.

the HR diagram as the one presented in Paper V. With respect to the model of Paper V, the MS lifetime is increased by about $7 \%$, while the helium-burning phase is decreased by the same amount. This results in part from the slightly increased initial hydrogen abundance in the present model $(X=0.705$ while in Paper V, $X=0.680)$. The mass loss during the MS phase has been significantly reduced in the present paper. Indeed, the present $20 M_{\odot}$ model at solar metallicity has lost during the MS phase a little more than one third of a solar mass, while the mass of the corresponding model presented in Paper V decreased by about one solar mass during the same period.

\section{The evolution of the internal rotation and meridional circulation}

The initial convergence of the internal $\Omega$-profile was discussed in Paper V and here the results are the same. The main point was that the $\Omega$-profile converges very quickly in about 1 to $2 \%$ of the MS lifetime towards an equilibrium profile. The same fast convergence occurs for the internal distribution of $U(r)$, the radial term of the vertical component of the meridional circulation. Then, during the MS phase $\Omega(r)$ decreases slowly keeping a small degree of differential rotation, which plays an essential role in the shear effects and the related transport mechanisms. We recall here the interesting result shown in Paper V that, in contradiction to the classical Eddington-Sweet theory, the velocity $U(r)$ does not depend very much on the rotational velocities.

Figure 1 shows the evolution of the $\Omega$-profile during the evolution on the MS of a $20 M_{\odot}$ star with $Z=0.004$

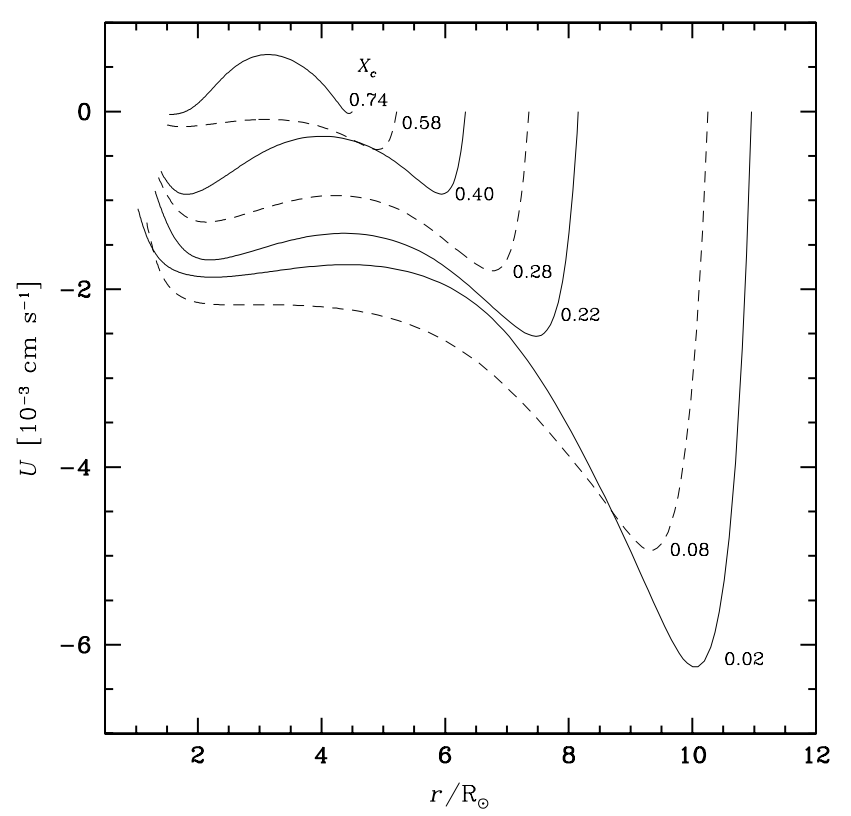

Fig. 2. Evolution of $U(r)$ the radial term of the vertical component of the velocity of meridional circulation for the same model as in Fig. 1. $X_{\mathrm{c}}$ is the hydrogen mass fraction at the center.

and an initial rotation velocity $v_{\text {ini }}=300 \mathrm{~km} \mathrm{~s}^{-1}$. Let us point out some differences with respect to the models at $Z=0.020$. For the same initial rotational velocities $v_{\text {ini }}$, at a given central $\mathrm{H}$-content $X_{\mathrm{c}}$, the values of $\Omega(r)$ are higher at $Z=0.004$. One first reason is rather trivial, i.e. because the radius is smaller at lower $Z$, the same $v_{\text {ini }}$ corresponds to a larger angular velocity $\Omega$. Indeed, the radius of a $20 M_{\odot}$ star at $Z=0.004$ is smaller, being equal to 0.8 of the radius of a same mass star at $Z=$ 0.020 . This ratio of 0.8 keeps closely the same during the whole MS phase. Another reason for the higher $\Omega(r)$ lies in the smaller mass loss rates, which lead to smaller losses of angular momentum, thus favouring a higher internal rotation at the end of the MS phase.

The internal gradients of $\Omega$ are higher at lower $Z$ due to the higher compactness of the star. This means that the outwards transport of chemical elements by shears will be favoured at lower $Z$. However, these larger shears do not destroy the $\Omega$-gradient, since quite generally shears are much less important than meridional circulation for transporting the angular momentum and shaping $\Omega(r)$.

Figure 2 shows the evolution of $U(r)$ in the same model. $U(r)$ is initially positive in the interior, but progressively the fraction of the star where $U(r)$ is negative is growing. This is due to the Gratton-Öpik term in Eq. (2), which favours a negative $U(r)$ in the outer layers, when the density decreases. This negative velocity causes an outward transport of the angular momentum, as well as the shears. Due to the higher density in the envelope at $Z=0.004$, the Gratton-Öpik term is less important and the values of $|U(r)|$ are smaller than at a solar composition. However, this is partly compensated by the smaller stellar radius which reduces the characteristic 


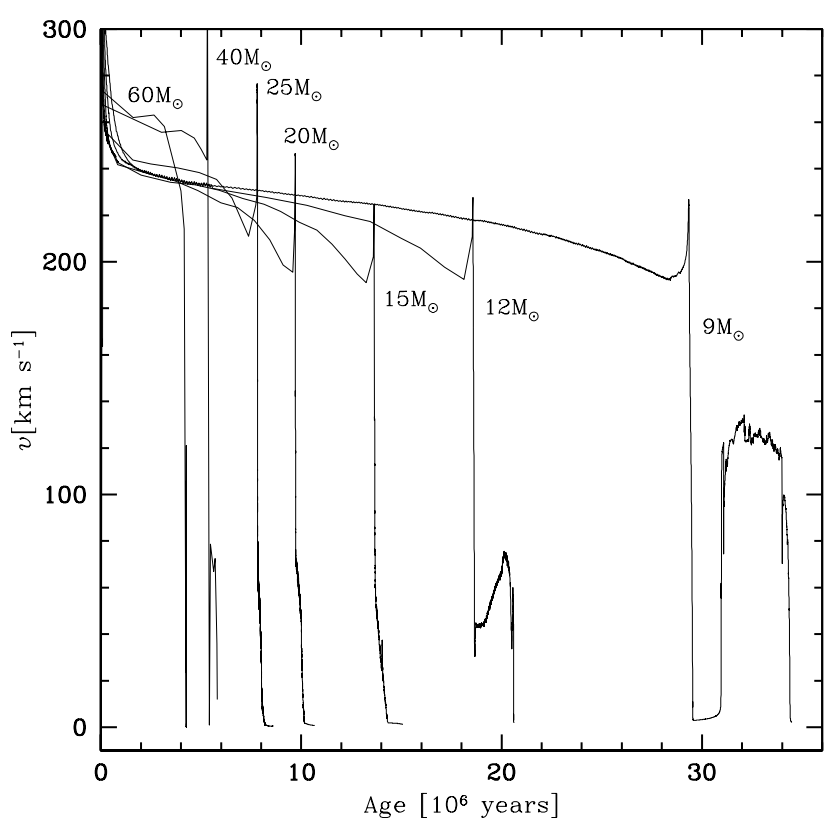

Fig. 3. Evolution of the surface equatorial velocity as a function of time for stars of different initial masses with $v_{\text {ini }}=$ $300 \mathrm{~km} \mathrm{~s}^{-1}$ and $Z=0.004$.

time for transport. In the model of $20 M_{\odot}$ at $Z=0.004$, we have the timescales $\tau_{\text {circ }} \sim R / U=1.5 \times 10^{7} \mathrm{yr}$ and $\tau_{\text {shear }} \sim R^{2} / D_{\text {shear }}=8.0 \times 10^{7}$ yr. This confirms that the circulation $U(r)$ is, as for models at $Z=0.02$, more important than the shears for transporting the angular momentum. In addition, we see that $\tau_{\text {circ }}$ is of the same order as the MS lifetime of $9.7 \times 10^{6} \mathrm{yr}$, which allows a significant transport to occur.

\section{The evolution of the surface rotation velocities}

The evolution of the rotational velocities $v$ at the stellar surface is shown in Fig. 3. We notice that for all masses considered here, $v$ remains almost constant until the end of the MS phase. Starting from an initial velocity $v_{\text {ini }}=300 \mathrm{~km} \mathrm{~s}^{-1}$ on the ZAMS, the initial convergence brings $v$ to about $250\left( \pm 10 \mathrm{~km} \mathrm{~s}^{-1}\right)$ and these values decrease to about $200 \mathrm{~km} \mathrm{~s}^{-1}$ for stars in the range of 9 to $25 M_{\odot}$ at the end of the MS phase. This behaviour is very different from that found for models with $Z=0.02$ (Paper $\mathrm{V}$ ), where the values of $v$ for the large masses decreased drastically (a value as low as $30 \mathrm{~km} \mathrm{~s}^{-1}$ was found for the $60 M_{\odot}$ star at the end of the MS). The reason for the decrease of $v$ at $Z=0.02$ was the large mass loss rate $\dot{M}$, while here at $Z=0.004$ the $\dot{M}$-rates are much smaller and the stars retain most of their angular momentum. This behaviour is reminiscent of that found in models with solid body rotation by Sackmann \& Anand (1970) and by Langer (1997, 1998).

Figure 4 shows the evolution of the fraction of the critical angular velocities $\frac{\Omega}{\Omega_{\text {crit }}}$; these results are striking and potentially very important. For the model of $9 M_{\odot}$, this fraction remains almost constant, but for the higher masses, the ratio $\frac{\Omega}{\Omega_{\text {crit }}}$ grows a lot and may even reach

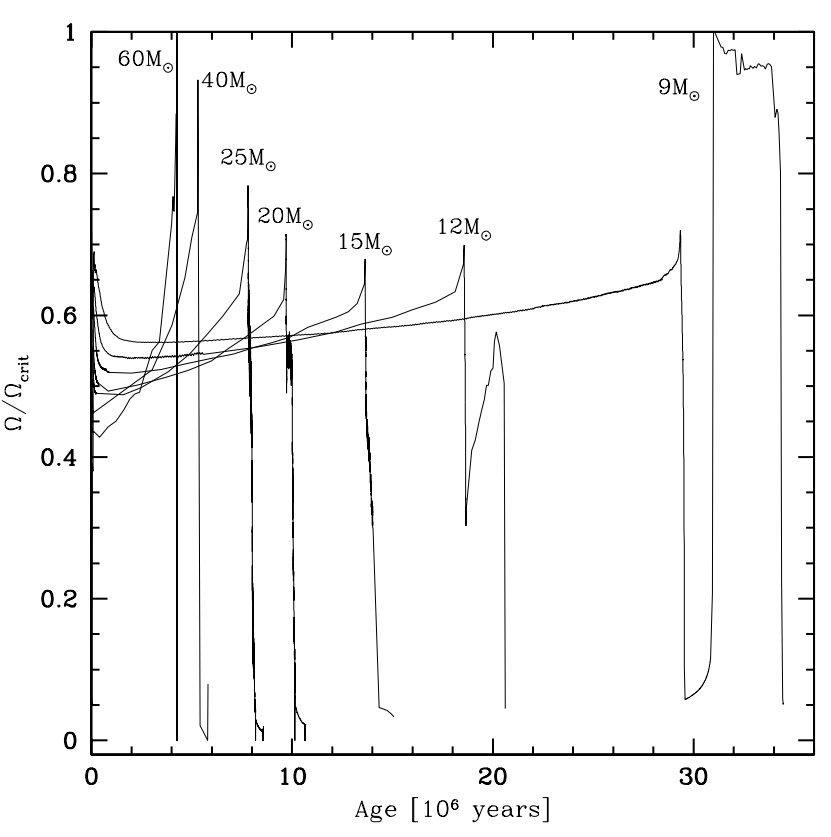

Fig. 4. Evolution of the ratio $\Omega / \Omega_{\text {crit }}$ of the angular velocity to the break-up angular velocity at the stellar surface for stars of different masses at $Z=0.004$.

1.0 during the overall contraction phase at the end of the MS phase. This is the opposite of the results of the models with $Z=0.02$, where the fraction $\frac{\Omega}{\Omega_{\text {rit }}}$ strongly decreased for the largest masses due to their high mass loss, which removed a huge amount of angular momentum. In the present models at $Z=0.004$, mass loss is insufficient to remove enough angular momentum.

We recall that, under the hypothesis of local conservation of angular momentum, the ratio $\frac{\Omega}{\Omega_{\text {crit }}}$ would decrease for a growing radius. Thus, the growth of $\frac{\Omega}{\Omega_{\text {crit }}}$ for the largest masses in Fig. 4 results essentially from the larger outwards transport of angular momentum by circulation. Indeed, we notice that $U(r)$ in a $40 M_{\odot}$ with $Z=0.004$ near the end of the MS reaches $-6 \times 10^{-2} \mathrm{~cm} \mathrm{~s}^{-1}$, compared to $-0.6 \times 10^{-2} \mathrm{~cm} \mathrm{~s}^{-1}$ in the $20 M_{\odot}$ illustrated in Fig. 2. The large negative velocities in the $40 M_{\odot}$ model at $Z=0.004$ transport more angular momentum outwards and thus explain the rise of $\frac{\Omega}{\Omega_{\text {crit }}}$ at the surface for large masses in Fig. 4.

Now, why is $U(r)$ more negative in larger masses? From Eq. (1) we see that for given values of $\left(\nabla_{\mathrm{ad}}-\nabla_{\mathrm{rad}}\right)$ and $\nabla_{\mu}, U(r)$ behaves essentially like

$U(r) \sim \frac{L}{\beta g M}\left[1-\frac{\Omega^{2}}{2 \pi G \bar{\rho}}\right]\left(\frac{\Omega^{2} r^{3}}{G M}\right)$.

For increasing stellar masses, we have a smaller value of the gravity $g$, a smaller value of $\beta$ the ratio of $P_{\text {gas }}$ to the total pressure $P$, a larger $L / M$ and a smaller density. All these differences contribute to make the values of $U(r)$ more negative. Together with the smaller mass loss, these are the physical reasons for the growth of the surface ratios $\frac{\Omega}{\Omega_{\text {crit }}}$ with the stellar masses. These results suggest that, if the initial distributions of the rotational velocities do not 


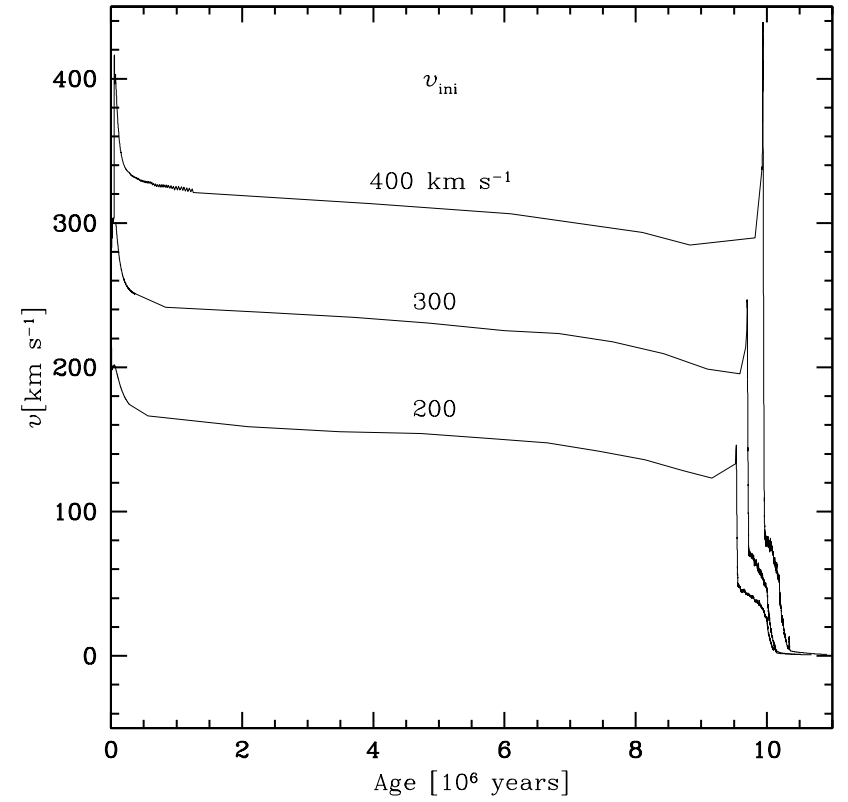

Fig. 5. Evolution of the surface equatorial velocity as a function of time for $20 M_{\odot}$ stars at $Z=0.004$ with different initial velocities.

have a higher fraction of slow rotators at lower metallicities (a point which is still unknown), then we may expect that at lower $Z$, as in the $S M C$, a much larger fraction of $O_{-}$and $B$-type stars are close to break-up velocities. This suggests that rotation is not necessarily a secondary parameter for the evolution of massive stars at low $Z$.

Figure 5 shows the evolution of the surface rotational velocities $v$ as a function of time for different initial velocities in the case of the model of $20 M_{\odot}$ at $Z=0.004$. We notice the rather parallel behaviour of the evolution of $v$. This is rather different from the models at $Z=0.02$, which showed a certain degree of convergence of $v$ at the end of the MS, due to the large mass loss rates.

With some necessary reservations, let us now make a few speculations. For zero metallicity models, the MS phase is shifted by 0.27 dex in $T_{\text {eff }}$ with respect to the models at solar composition, which means radii smaller by a factor of 3.4 at a given luminosity for $Z=0$. For the models at $Z=0.004$, the blue shift amounts only to 0.04 dex, implying a radius smaller by a factor of 1.2 . Thus, we see that the degree of compactness at very low $Z$ is much stronger. Thus, the models at $Z=0$ will likely amplify the behaviour shown by the models at $Z=0.004$, due to their higher compactness and their smaller mass loss rates. Thus, we may wonder whether a large fraction, if not most, massive stars at very low $Z$ do not reach break-up velocities during their MS phases. The observations of a much higher fraction of Be-stars (cf. Maeder et al. 1999) in the LMC and SMC compared to the Milky Way tend to support this view.

Finally, we notice in Fig. 4 the occurence of the socalled spin-up effect in the $9 M_{\odot}$ on the blue loop: as the star is contracting, its rotation strongly accelerates. This effect has been described by Heger \& Langer (1998). The envelope accelerates its rotation, due to both its contraction and the fact that the convective zone is receding, as shown by Heger \& Langer.

\section{Models with zero rotation}

The evolutionary tracks and the lifetimes of the nonrotating tracks at $Z=0.004$ are presented in Figs. 6 and 7 and in Table 1. With respect to the previous Geneva grids of stellar models at $Z=0.004$ (Charbonnel et al. 1993; Meynet et al. 1994), those presented here show the following main differences: the MS width is reduced, typically at $\log T_{\text {eff }}=4.4$, the width in $\Delta \log L / L_{\odot}$ is decreased by 0.34 dex; for initial mass star models below $40 M_{\odot}$, the MS lifetimes are shorter by about $11 \%$ for the $9 M_{\odot}$ model and by $1.5 \%$ for the $40 M_{\odot}$ model; the ratios of the $\mathrm{He}-$ to the $\mathrm{H}$-burning lifetimes are greater by about a factor two for the $9 M_{\odot}$ model and by $7 \%$ for the $40 M_{\odot}$ model. All these effects are quite consistent with the fact that, in the present work, we did not include any overshooting (see e.g. Bertelli et al. 1985; Maeder \& Meynet 1989; Chin \& Stothers 1990; Langer \& Maeder 1995 for detailed discussions of the effects of overshooting on the stellar models). For initial masses superior to about $60 M_{\odot}$, the effects of the stellar winds become very important and dominate the effects due to a change of the criterion for convection. Qualitatively similar differences are obtained when the present results are compared with the stellar models at $Z=0.004$ computed with overshooting by Fagotto et al. (1994) and Claret \& Gimenez (1998).

The models with initial masses between 10 and $12.5 M_{\odot}$ are peculiar, they show a behaviour in the HR diagram intermediate between the cases of stars presenting a well developed blue loop (as the 9 and the $10 M_{\odot}$ models in Fig. 7) and the case of more massive stars which do not produce any blue loop, but begin to burn their helium in their core at a high effective temperature while they cross the HR diagram for the first time (as the 12.5 and $13 M_{\odot}$ in Fig. 7). The models in the transition mass range (i.e. the 10.5, 11 and $12 M_{\odot}$ models in Fig. 7) present a "partial blue loop" which starts at a high effective temperature and then the models spend nearly their whole helium burning phase in the blue (see Sect. 7). Such a behaviour has also been found by Charbonnel et al. (1993) for their $15 M_{\odot}$ model and by Claret \& Gimenez (1998) for stars with initial masses between about 16 and $30 M_{\odot}$. In this last case, the exact mass range depends on the adopted value for the initial abundance of helium. These results seem to indicate that an extension of the $\mathrm{H}$-burning core, e.g. by overshooting, shifts the transition range to higher initial masses, which is quite consistent. The models by Fagotto et al. (1994) do not show such a behaviour between 15 and $30 M_{\odot}$. This may be due to the fact that these models were computed with a much greater overshooting parameter than the two grids mentioned above. 


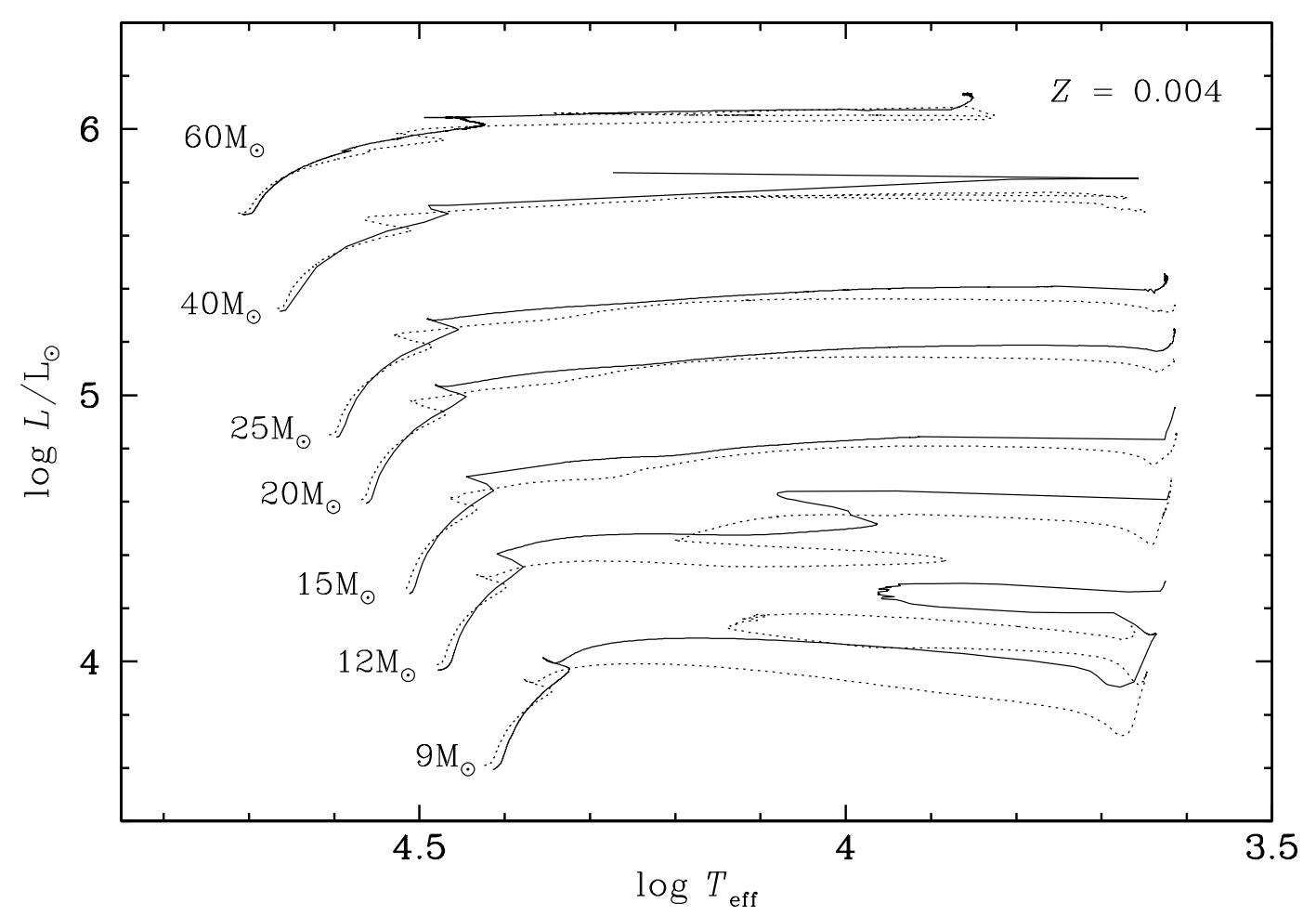

Fig. 6. Evolutionary tracks for non-rotating (dotted lines) and rotating (continuous lines) models for a metallicity $Z=0.004$. The rotating models have an initial velocity $v_{\text {ini }}$ of $300 \mathrm{~km} \mathrm{~s}^{-1}$. For the rotating $60 M_{\odot}$ model, only part of the evolution is plotted.

\section{HR diagram, mass-luminosity relation and lifetimes}

\subsection{A brief review of the effects of rotation on the stellar models}

Many effects induced by rotation at solar metallicity are also found here at lower metallicity. These effects have been described in detail by Heger et al. (2000), Maeder \& Meynet (2000a) and Meynet \& Maeder (2000). Thus we will be very brief here. Rotation modifies the evolutionary tracks in the HR diagram through the following main physical effects: rotation lowers the effective gravity $g_{\text {eff }}$; it enlarges the convective cores and smoothes the chemical gradients in the radiative zones (the main effect); it enhances the mass loss rates; it produces atmospheric distortions. Since the star has lost its spherical symmetry, the tracks may also change in appearance depending on the angle of view. Here we suppose an average angle of view, as was done in Paper V.

As a consequence of these effects, depending on the degree of mixing (see below), the evolutionary tracks on the MS are extended towards lower effective temperatures (as would do a moderate overshooting) and/or are made overluminous. The evolution towards the red supergiant stage is favoured (see Sect. 7), as well as the evolution into the WR phase. Moreover, as will be discussed below, the MS lifetimes are increased and the surface abundances are modified.
Since there are different rotational velocities, a star of given initial mass and metallicity can follow different tracks corresponding to various initial rotational velocities. To minimize the number of tracks to be computed, we have to choose a value of the initial velocity which produces an average velocity during the MS not too far from the observed value. The problem here is that one does not know what this average rotational velocity is for OB stars at the metallicity of the Small Magellanic Cloud (SMC). In the absence of such an information, we adopt here the same initial rotational velocity as in our grid at $Z=0.020$, namely the value $v_{\text {ini }}=300 \mathrm{~km} \mathrm{~s}^{-1}$. Defining a mean equatorial velocity $\bar{v}$ during the MS as in Meynet \& Maeder (2000), such a value for $v_{\text {ini }}$ corresponds to values of $\bar{v}$ between 220 and $260 \mathrm{~km} \mathrm{~s}^{-1}$. These values are close to the average rotational velocities observed for OBV type stars at solar metallicity, which are between 200$250 \mathrm{~km} \mathrm{~s}^{-1}$.

\subsection{The HR diagram}

Figure 6 shows the evolutionary tracks of non-rotating and rotating stellar models for initial masses between 9 and $60 M_{\odot}$. One sees that the MS width is increased, as would result from a moderate overshoot. Let us recall here that two counteracting effects of rotation affect the extension of the MS band (see Paper V). On one hand, rotational mixing brings fresh $\mathrm{H}$-fuel into the convective core, slowing down its decrease in mass during the MS. This effect produces a more massive $\mathrm{He}-$ core at the end 
of the $\mathrm{H}$-burning phase and this favours the extension of the tracks towards lower effective temperatures. On the other hand, rotational mixing transports helium and other $\mathrm{H}$-burning products (essentially nitrogen) into the radiative envelope. The He-enrichment lowers the opacity. This contributes to the enhancement of the stellar luminosity and favours a blueward track. Clearly here, the first effect dominates over the second one.

In this context, it is interesting to recall that, due to the account of horizontal turbulence in the present models, the mixing of the chemical elements by the shear has been effectively reduced in the regions of steep $\mu$-gradient with respect to Paper V (see Sect. 2). This favours, for a given initial rotational velocity, an extension of the MS towards lower effective temperatures. Indeed when rotational mixing is decreased, either as a consequence of the reduction of the initial velocity or by a reduction of the shear diffusion coefficient as in the present models, the time required for helium mixing in the whole radiative envelope is considerably increased, while the time for hydrogen to migrate into the convective core, although it is also increased, remains nevertheless relatively small since hydrogen just needs to diffuse through a small amount of mass to reach the convective core (see Meynet \& Maeder 2000). Thus, some increase in the size of the core results, while the effect on the helium abundance in the envelope is not significant. The same kind of effect can be seen in the models by Talon et al. (1997). Of course the $\mu^{-}$ gradients are strong near the core and can slow down the diffusion process mentioned above, but on the other hand, the efficiency of the diffusion of hydrogen will also increase with the increasing $\mathrm{H}$-abundance gradient at the border of the core. These are the various reasons why the numerical models show that, for a moderate rotational mixing, the effect of rotation on the convective core mass overcomes the effect of helium diffusion in the envelope.

Let us recall that some core overshooting was needed in stellar models in order to reproduce the observed MS width (see Maeder \& Mermilliod 1981; Maeder \& Meynet 1989). Typically, the width of the MS band in $\Delta \log L / L_{\odot}$ at $\log T_{\text {eff }}=4.4$ obtained from the present non-rotating models is 0.85 , while a value of about 1.20 is required to reproduce the observation. Such a width can be reproduced with a moderate amount of overshooting (see Schaller et al. 1992).

Rotation as we just saw above produces also a wider MS. Indeed, the width of the MS band in $\Delta \log L / L_{\odot}$ at $\log T_{\text {eff }}=4.4$ for the rotating models presented in Fig. 6 is slightly superior to 1 . This comparison shows that if the $v_{\text {ini }}=300 \mathrm{~km} \mathrm{~s}^{-1}$ models are well representative of the average case, then rotation alone might account for about half of the MS widening required by the observations. This is only a rough estimate. The MS extension is different for different initial rotational velocities (see Fig. 8), it will also be slightly different depending on the angle of view of the stars. Therefore the evaluation of the contributions of rotation and overshooting to the widening of the MS band requires the computations of numerous

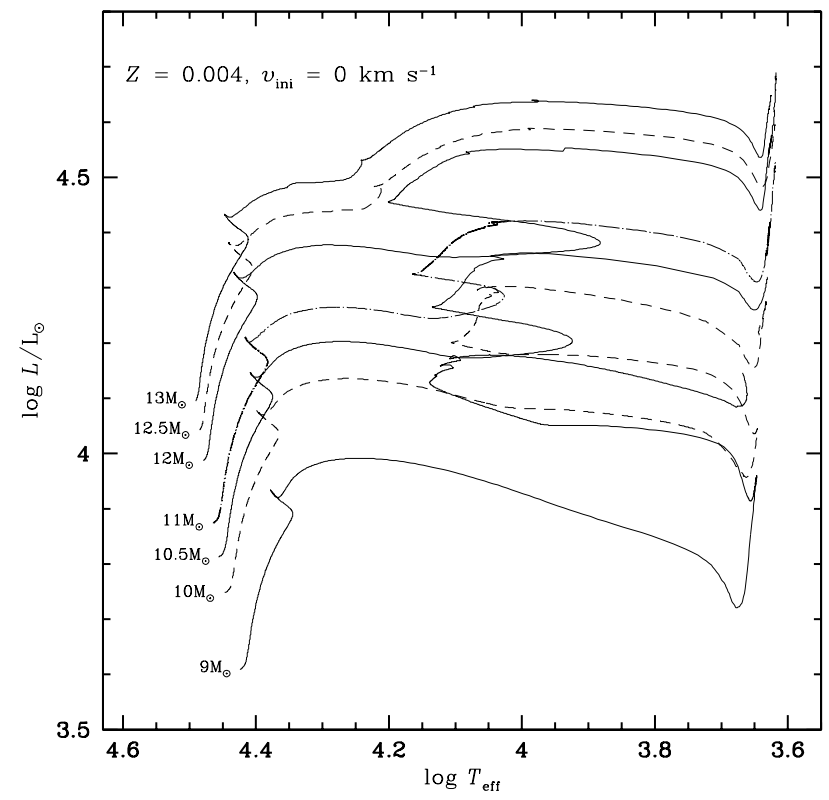

Fig. 7. Evolutionary tracks for non-rotating stellar models at $Z=0.004$ in the transition region between stars with and without blue loops. The initial masses are indicated in solar masses.

models for various initial masses and rotational velocities so that detailed population synthesis models can be performed. However, we can say with some confidence that rotation decreases by about a factor of 2 the amount of overshooting needed to reproduce the observed MS width, as was already proposed by Talon et al. (1997).

One striking difference between non-rotating and rotating models after the MS concerns the fraction of the helium burning phase spent as a red supergiant. This point will be discussed in detail in the next section. Let us also mention here that rotation shortens the blue loops of the $9 M_{\odot}$ model. This is a consequence of the more massive helium cores existing at the end of the $\mathrm{H}$-burning phase in rotating models, as well as of the addition of helium near the H-burning shell (cf. Sect. 7.2). As in the case of the $9 M_{\odot}$ model and for the same reason, rotation reduces the extension of the "partial" blue loop associated with the $12 M_{\odot}$ model.

For the rotational velocities considered here, no model enters the WR phase during the core $\mathrm{H}$-burning phase. But after the MS phase, the rotating $40 M_{\odot}$ model enters the WR phase while its non-rotating counterpart does not. This illustrates the fact that rotation decreases the minimum initial mass for a single star to become a WR star (Maeder 1987; Fliegner \& Langer 1995; Maeder \& Meynet 2000a; Meynet 2000). We shall not develop this point further here since it will be the subject of a forthcoming paper. 


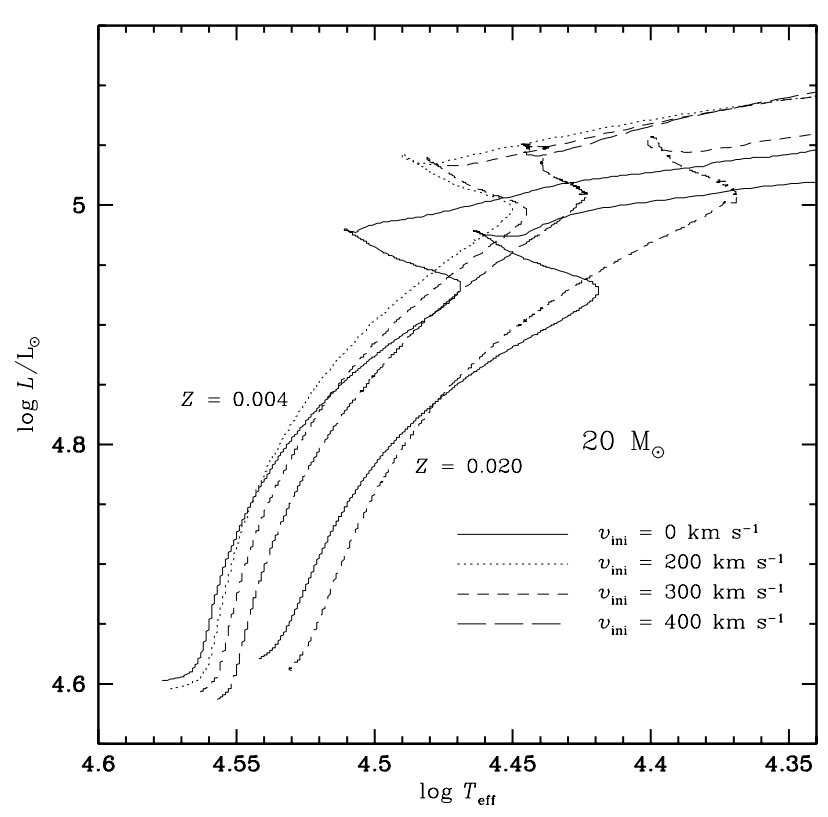

Fig. 8. Evolutionary tracks for rotating $20 M_{\odot}$ models with different initial velocities and various initial metallicities. The initial velocities $v_{\text {ini }}$ are indicated. See Table 1 for more details on the models at $Z=0.004$.

\subsection{Differences between rotating tracks at $Z=0.004$ and 0.020}

Since the physics has been improved with respect to Paper V (see Sect. 2), we cannot directly compare the solar tracks of Paper $\mathrm{V}$ with the present ones. Therefore we computed one $20 M_{\odot}$ model at solar metallicity with and without rotation with exactly the same physics as in the present paper. These models are plotted together with the $20 M_{\odot}$ models at $Z=0.004$ in Fig. 8 .

From this figure, one sees that rotation at low metallicity has similar effects than at solar metallicity. For instance, the increase due to rotation of the He-core masses at the end of the $\mathrm{H}$-burning phase is similar at $Z=0.020$ and $Z=0.004$. Typically, for the $20 M_{\odot}$ models shown in Fig. 8, one has that in the non-rotating models, both at $Z=0.020$ and $Z=0.004$, the helium cores contain $26 \%$ of the total mass at the end of the MS phase. In the rotating models with $v_{\text {ini }}=300 \mathrm{~km} \mathrm{~s}^{-1}$, this mass fraction is enhanced up to values between $30-32 \%$.

Due to the distribution of the initial velocities and of the orientations of the angles of view, rotation induces some scatter of the luminosities and effective temperatures at the end of the MS phase (see Paper V). One observes from Fig. 8 that, at low metallicity, for a given initial velocity, the extension towards lower effective temperatures due to rotation is slightly reduced (compare the tracks for $v_{\text {ini }}=300 \mathrm{~km} \mathrm{~s}^{-1}$ ). Thus, at low $Z$, our models show that, if the initial rotation velocities are distributed in the same manner as at solar metallicity, the scatter of the effective temperatures and luminosities at the end of the MS will be reduced.

\subsection{Masses and mass-luminosity relations}

When rotation increases, the actual masses at the end of both the MS and the He-burning phases become smaller (cf. Table 1). Typically the quantity of mass lost by stellar winds during the MS is enhanced by $45-75 \%$ in rotating models with $v_{\text {ini }}=300 \mathrm{~km} \mathrm{~s}^{-1}$. Similar enhancements were found at solar metallicity (see Paper V). The increase is due mainly to the direct effect of rotation on the mass loss rates (cf. Eq. (5)). The higher luminosities reached by the rotating models and their longer MS lifetimes also contribute somewhat to produce smaller final masses.

Rotation makes the star overluminous for their actual masses. Typically for $v_{\text {ini }}=300 \mathrm{~km} \mathrm{~s}^{-1}$, the luminosity vs. mass ratios at the end of the MS are increased by $15-22 \%$. It is interesting to mention here that even if the rotating and non-rotating tracks in the $\log L / L_{\odot}$ vs. $\log T_{\text {eff }}$ plane are very similar, they may present large differences in the $\log g_{\text {eff }}$ vs. $\log T_{\text {eff }}$ plane where $g_{\text {eff }}$ is estimated at an average orientation angle as in Paper V. The large difference in $\log g_{\text {eff }}$ for similar masses, luminosities and effective temperatures comes from the fact that the effective gravity of the rotating model differs from the gravity of the non-rotating model by an amount equal to the centrifugal acceleration. Typically, the two $40 M_{\odot}$ tracks plotted in Fig. 6 which, at $\log T_{\text {eff }}=4.4$, differ by only $\sim 0.04 \mathrm{dex}$ in $\log L / L_{\odot}$, show important differences in the $\log g_{\text {eff }}$ vs. $\log T_{\text {eff }}$ plane. For instance, the rotating $40 M_{\odot}$ track overlaps the non-rotating $60 M_{\odot}$ model in this plane. Therefore, one could expect that the attribution of a mass to an observed star position in the $\log g_{\text {eff }}$ vs. $\log T_{\text {eff }}$ plane is very rotation dependent. The use of non rotating tracks would overestimate the mass (in the example above by $50 \%$ ), and this might be a cause of the well known problem of the mass discrepancy (see e.g. Herrero et al. 2000). Let us note that in practice the effective gravity and the other physical quantities are derived from the spectral lines, which shapes and equivalent widths are also affected by rotation.

\subsection{Lifetimes}

Table 1 presents some properties of the models. Columns 1 and 2 give the initial mass and the initial velocity $v_{\text {ini }}$ respectively. The mean equatorial rotational velocity $\bar{v}$ during the MS phase is indicated in Col. 3. The H-burning lifetimes $t_{\mathrm{H}}$, the masses $M$, the equatorial velocities $v$, the helium surface abundance $Y_{\mathrm{s}}$ and the surface ratios $\mathrm{N} / \mathrm{C}$ and $\mathrm{N} / \mathrm{O}$ at the end of the $\mathrm{H}$-burning phase and normalized to their initial values are given in Cols. 4 to 9 . The Cols. 10 to 13 present some properties of the models when $\log T_{\text {eff }}=4.0$ during the crossing of the Hertzsprung-Russel diagram, or when the star enters into the WR phase (for the rotating $40 M_{\odot}$ models and the non-rotating $60 M_{\odot}$ model), or at the bluest point on the blue loop (for the models with $M \leq 12 M_{\odot}$ ). The Cols. 14 to 19 present some characteristics of the stellar models at 
Table 1. Properties of the stellar models at the end of the H-burning phase, at $\log T_{\text {eff }}=4.0$ (see text) and at the end of the He-burning phase. The masses are in solar mass, the velocities in $\mathrm{km} \mathrm{s}^{-1}$, the lifetimes in million years and the helium abundance in mass fraction. The abundance ratios are normalized to their initial value.

\begin{tabular}{|c|c|c|c|c|c|c|c|c|c|c|c|c|c|c|c|c|c|c|}
\hline \multirow[b]{2}{*}{$M$} & \multirow[b]{2}{*}{$v_{\text {ini }}$} & \multirow[b]{2}{*}{$\bar{v}$} & \multicolumn{6}{|c|}{ End of $\mathrm{H}$-burning } & \multicolumn{4}{|c|}{$\log T_{\text {eff }}=4.0$} & \multicolumn{6}{|c|}{ End of He-burning } \\
\hline & & & $t_{\mathrm{H}}$ & $M$ & $v$ & $Y_{\mathrm{s}}$ & $\mathrm{N} / \mathrm{C}$ & $\mathrm{N} / \mathrm{O}$ & $v$ & $Y_{\mathrm{S}}$ & $\mathrm{N} / \mathrm{C}$ & $\mathrm{N} / \mathrm{O}$ & $t_{\mathrm{He}}$ & $M$ & $v$ & $Y_{\mathrm{s}}$ & $\mathrm{N} / \mathrm{C}$ & $\mathrm{N} / \mathrm{O}$ \\
\hline 60 & $\begin{array}{c}0 \\
300\end{array}$ & $\begin{array}{c}0 \\
259\end{array}$ & $\begin{array}{l}3.951 \\
4.232\end{array}$ & $\begin{array}{l}57.709 \\
56.415\end{array}$ & $\begin{array}{c}0 \\
307\end{array}$ & $\begin{array}{l}0.24 \\
0.29\end{array}$ & $\begin{array}{l}1.00 \\
11.3\end{array}$ & $\begin{array}{l}1.00 \\
9.50\end{array}$ & 0 & 0.57 & 127 & 267 & 0.345 & 41.733 & 0 & 0.60 & 185 & 198 \\
\hline 40 & $\begin{array}{c}0 \\
300\end{array}$ & $\begin{array}{c}0 \\
257\end{array}$ & $\begin{array}{l}4.924 \\
5.312\end{array}$ & $\begin{array}{l}39.066 \\
38.650\end{array}$ & $\begin{array}{c}0 \\
332\end{array}$ & $\begin{array}{l}0.24 \\
0.25\end{array}$ & $\begin{array}{l}1.00 \\
7.26\end{array}$ & $\begin{array}{l}1.00 \\
4.75\end{array}$ & $\begin{array}{c}0 \\
81\end{array}$ & $\begin{array}{l}0.24 \\
0.60\end{array}$ & $\begin{array}{l}1.00 \\
113\end{array}$ & $\begin{array}{l}1.00 \\
68.2\end{array}$ & $\begin{array}{l}0.460 \\
0.496\end{array}$ & $\begin{array}{l}30.222 \\
20.481\end{array}$ & $\begin{array}{c}0 \\
21\end{array}$ & $\begin{array}{l}0.49 \\
0.73\end{array}$ & $\begin{array}{l}145 \\
203\end{array}$ & $\begin{array}{l}66.0 \\
595\end{array}$ \\
\hline 25 & $\begin{array}{c}0 \\
300\end{array}$ & $\begin{array}{c}0 \\
239\end{array}$ & $\begin{array}{l}7.196 \\
7.809\end{array}$ & $\begin{array}{l}24.689 \\
24.557\end{array}$ & $\begin{array}{c}0 \\
274\end{array}$ & $\begin{array}{l}0.24 \\
0.24\end{array}$ & $\begin{array}{l}1.00 \\
4.61\end{array}$ & $\begin{array}{l}1.00 \\
3.25\end{array}$ & $\begin{array}{c}0 \\
56\end{array}$ & $\begin{array}{l}0.24 \\
0.25\end{array}$ & $\begin{array}{l}1.00 \\
5.42\end{array}$ & $\begin{array}{l}1.00 \\
3.75\end{array}$ & $\begin{array}{l}0.806 \\
0.752\end{array}$ & $\begin{array}{l}24.322 \\
20.015\end{array}$ & $\begin{array}{c}0 \\
0.6\end{array}$ & $\begin{array}{l}0.24 \\
0.39\end{array}$ & $\begin{array}{l}1.00 \\
29.5\end{array}$ & $\begin{array}{l}1.00 \\
18.0\end{array}$ \\
\hline 20 & $\begin{array}{c}0 \\
200 \\
300 \\
400\end{array}$ & $\begin{array}{c}0 \\
152 \\
229 \\
311\end{array}$ & $\begin{array}{l}8.736 \\
9.533 \\
9.700 \\
9.940\end{array}$ & $\begin{array}{l}19.833 \\
19.777 \\
19.750 \\
19.683\end{array}$ & $\begin{array}{c}0 \\
146 \\
244 \\
429\end{array}$ & $\begin{array}{l}0.24 \\
0.24 \\
0.24 \\
0.24\end{array}$ & $\begin{array}{l}1.00 \\
2.94 \\
4.94 \\
6.39\end{array}$ & $\begin{array}{l}1.00 \\
2.25 \\
3.25 \\
3.75\end{array}$ & $\begin{array}{c}0 \\
33 \\
53 \\
65\end{array}$ & $\begin{array}{l}0.24 \\
0.24 \\
0.24 \\
0.25\end{array}$ & $\begin{array}{l}1.00 \\
3.52 \\
5.58 \\
6.84\end{array}$ & $\begin{array}{l}1.00 \\
2.50 \\
3.50 \\
4.00\end{array}$ & $\begin{array}{l}1.007 \\
0.963 \\
0.960 \\
0.952\end{array}$ & $\begin{array}{l}19.690 \\
18.376 \\
18.039 \\
17.758\end{array}$ & $\begin{array}{c}0 \\
1.2 \\
0.8 \\
1.0\end{array}$ & $\begin{array}{l}0.24 \\
0.29 \\
0.31 \\
0.33\end{array}$ & $\begin{array}{l}1.65 \\
13.2 \\
17.0 \\
20.4\end{array}$ & $\begin{array}{l}1.25 \\
8.00 \\
9.50 \\
11.0\end{array}$ \\
\hline 15 & $\begin{array}{c}0 \\
300\end{array}$ & $\begin{array}{c}0 \\
225\end{array}$ & $\begin{array}{l}12.158 \\
13.641\end{array}$ & $\begin{array}{l}14.910 \\
14.854\end{array}$ & $\begin{array}{c}0 \\
226\end{array}$ & $\begin{array}{l}0.24 \\
0.24\end{array}$ & $\begin{array}{l}1.00 \\
6.16\end{array}$ & $\begin{array}{l}1.00 \\
3.50\end{array}$ & $\begin{array}{c}0 \\
40\end{array}$ & $\begin{array}{l}0.24 \\
0.24\end{array}$ & $\begin{array}{l}1.00 \\
6.68\end{array}$ & $\begin{array}{l}1.00 \\
3.75\end{array}$ & $\begin{array}{l}1.515 \\
1.389\end{array}$ & $\begin{array}{l}14.686 \\
14.124\end{array}$ & $\begin{array}{c}0 \\
1.2\end{array}$ & $\begin{array}{l}0.25 \\
0.29\end{array}$ & $\begin{array}{l}5.00 \\
18.0\end{array}$ & $\begin{array}{l}3.00 \\
8.75\end{array}$ \\
\hline 12 & $\begin{array}{c}0 \\
300\end{array}$ & $\begin{array}{c}0 \\
225\end{array}$ & $\begin{array}{l}16.560 \\
18.568\end{array}$ & $\begin{array}{l}11.969 \\
11.950\end{array}$ & $\begin{array}{c}0 \\
228\end{array}$ & $\begin{array}{l}0.24 \\
0.24\end{array}$ & $\begin{array}{l}1.00 \\
5.06\end{array}$ & $\begin{array}{l}1.00 \\
3.00\end{array}$ & $\begin{array}{c}0 \\
74\end{array}$ & $\begin{array}{l}0.24 \\
0.28\end{array}$ & $\begin{array}{l}1.00 \\
16.5\end{array}$ & $\begin{array}{l}1.00 \\
7.75\end{array}$ & $\begin{array}{l}2.810 \\
1.978\end{array}$ & $\begin{array}{l}11.902 \\
11.807\end{array}$ & $\begin{array}{c}0 \\
1.9\end{array}$ & $\begin{array}{l}0.24 \\
0.29\end{array}$ & $\begin{array}{l}2.84 \\
18.6\end{array}$ & $\begin{array}{l}1.75 \\
8.50\end{array}$ \\
\hline 9 & $\begin{array}{c}0 \\
300\end{array}$ & $\begin{array}{c}0 \\
222\end{array}$ & $\begin{array}{l}25.911 \\
29.349\end{array}$ & $\begin{array}{l}8.996 \\
8.993\end{array}$ & $\begin{array}{c}0 \\
225\end{array}$ & $\begin{array}{l}0.24 \\
0.24\end{array}$ & $\begin{array}{l}1.00 \\
4.23\end{array}$ & $\begin{array}{l}1.00 \\
2.50\end{array}$ & $\begin{array}{c}0 \\
127\end{array}$ & $\begin{array}{l}0.24 \\
0.25\end{array}$ & $\begin{array}{l}3.19 \\
9.81\end{array}$ & $\begin{array}{l}2.00 \\
5.00\end{array}$ & $\begin{array}{l}4.543 \\
4.927\end{array}$ & $\begin{array}{l}8.966 \\
8.542\end{array}$ & $\begin{array}{c}0 \\
2.2\end{array}$ & $\begin{array}{l}0.24 \\
0.25\end{array}$ & $\begin{array}{l}3.19 \\
10.1\end{array}$ & $\begin{array}{l}1.75 \\
5.25\end{array}$ \\
\hline
\end{tabular}

the end of the He-burning phase; $t_{\mathrm{He}}$ is the He-burning lifetime.

From Table 1 one sees that for $Z=0.004$ the MS lifetimes are increased by about $7-13 \%$ for the mass range between 9 and $60 M_{\odot}$ when $v_{\text {ini }}$ increases from 0 to $\sim 300 \mathrm{kms}^{-1}$. In general, the corresponding changes in the He-burning lifetimes are inferior to $10 \%$. As was the case at solar metallicity, the ratios $t_{\mathrm{He}} / t_{\mathrm{H}}$ of the $\mathrm{He}-$ to $\mathrm{H}$-burning lifetimes are only slightly decreased by rotation and remain around $9-17 \%$. At solar metallicity, the changes of the lifetimes due to rotation are quite similar. The rotating $20 M_{\odot}$ model with $v_{\text {ini }}=300 \mathrm{~km} \mathrm{~s}^{-1}$, at solar metallicity, has a MS lifetime increased by $14 \%$ with respect to the non-rotating model. At $Z=0.004$, the corresponding increase amounts to $11 \%$, which is not significantly different.

\section{The ratio of blue to red supergiants in the SMC}

\subsection{Model results}

The observed ratio $\mathrm{B} / \mathrm{R}$ of blue to red supergiants in the SMC cluster NGC 330 lies between 0.5 and 0.8 , according to the various sources discussed in Langer \& Maeder (1995). Not many new results have been obtained since then. New IR searches have revealed some AGB stars in the SMC (Zilstra et al. 1996) and ISO observations (Kucinskas et al. 2000) have led to the detection of an IR source in NGC 330, which may be a Be supergiant or a post AGB-star, but this does not change the statistics significantly. Notice that the definition of $B / R$ is not always the same, e.g. for Humphreys \& McElroy (1984), B means $\mathrm{O}, \mathrm{B}$ and $\mathrm{A}$-supergiants. Here, we strictly count in the $B / R$ ratio the $B$ star models from the end of the MS to type B9.5 I, which corresponds to $\log T_{\text {eff }}=3.99$ according to the calibration by Flower (1996). We count as red supergiants all star models below $\log T_{\text {eff }}=3.70$ since red supergiants in the SMC are not as red as in the Galaxy (Humphreys 1979). We note that the exact definition of this limit has no influence on the observed or theoretical $B / R$ ratios, since the evolution through types $F, G, K$ is always very fast.

As noted by Langer \& Maeder (1995), the current models (without rotation) with Schwarzschild's criterion predict no red supergiants in the SMC (cf. Schaller et al. 1992). This is also seen in Fig. 9 which illustrates for models of $20 M_{\odot}$ at $Z=0.004$ the variations of the $T_{\text {eff }}$ as a function of the fractional lifetime in the He-burning phase for different rotation velocities. For zero rotation, we see that the star only moves to the red supergiants at the very end of the He-burning phase, so that the $\mathrm{B} / \mathrm{R}$ ratio, with the definitions given above, is $\mathrm{B} / \mathrm{R} \simeq 47$. For average rotational velocities $\bar{v}$ during the $\mathrm{MS}, \bar{v}=152,229$ and $311 \mathrm{~km} \mathrm{~s}^{-1}$, one has respectively $\mathrm{B} / \mathrm{R}=1.11,0.43$ and 0.28 . Thus, the $B / R$ ratios are much smaller for higher initial rotation velocities, as rotation favours the formation of red supergiants and reduce the lifetime in the blue. We notice in particular that for $\bar{v}=200 \mathrm{~km} \mathrm{~s}^{-1}$, we have a $B / R$ ratio of about 0.6 well corresponding to the range of the observed values.

The $\mathrm{B} / \mathrm{R}$ ratios change with the stellar masses. Figure 10 shows for the models of 15,20 and $25 M_{\odot}$ the changes of $T_{\text {eff }}$ as a function of the fractional lifetimes in the He-burning phase for different rotation. For all masses, we notice that the non-rotating stars spend nearly the whole of their $\mathrm{He}$-phase as blue supergiants 


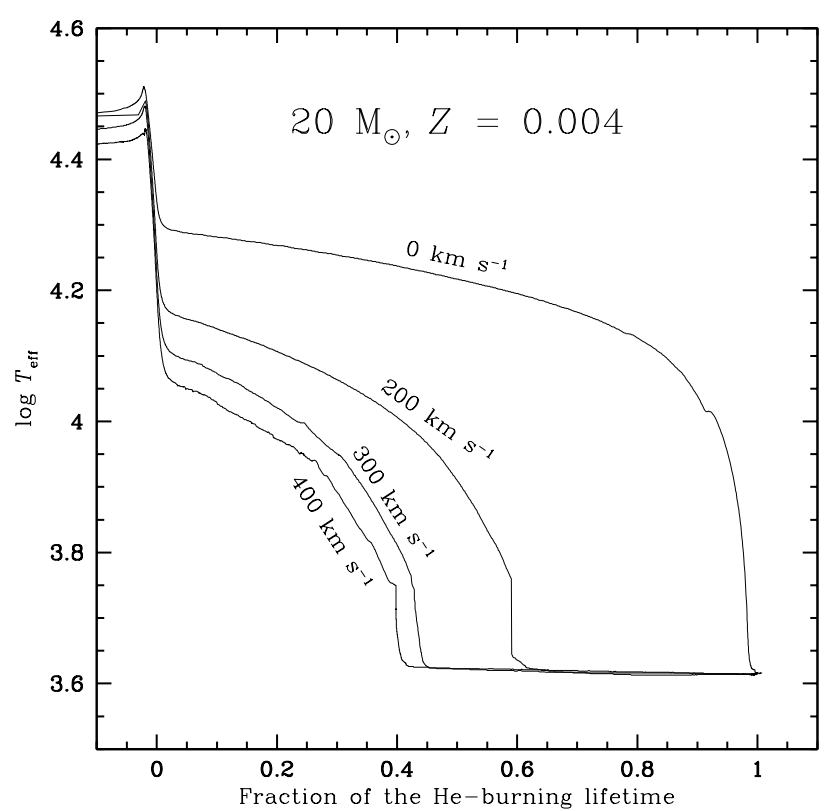

Fig. 9. Evolution of the $T_{\text {eff }}$ as a function of the fraction of the lifetime spent in the He-burning phase for $20 M_{\odot}$ stars with different initial velocities.

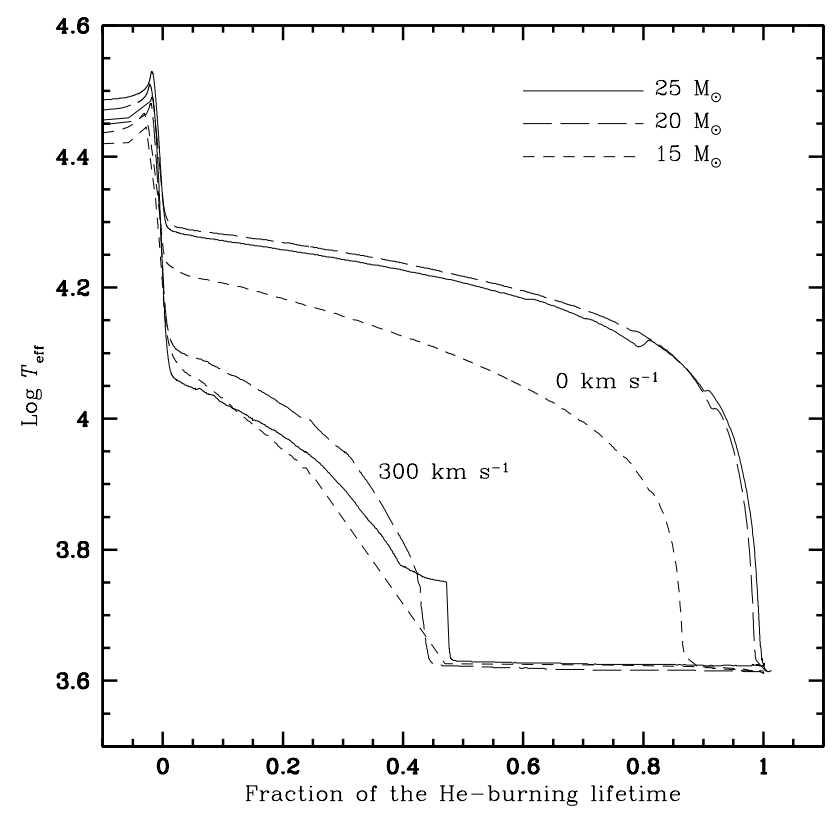

Fig. 10. Evolution of the $T_{\text {eff }}$ as a function of the fraction of the lifetime spent in the He-burning phase for 15, 20 and $25 M_{\odot}$ stars at $Z=0.004$ with $v_{\text {ini }}=0$ and $300 \mathrm{~km} \mathrm{~s}^{-1}$.

and almost none as red supergiants. For $v_{\text {ini }}=300 \mathrm{~km} \mathrm{~s}^{-1}$ (which corresponds to about $\bar{v}=220 \mathrm{~km} \mathrm{~s}^{-1}$ ), we notice a drastic decrease of the blue phase and a corresponding large increase of the red supergiant phase.

Figure 11 shows the same as Fig. 9 but for the models of 9 and $12 M_{\odot}$. These models mark the transition from the behaviour of massive stars, which move at various paces from blue to red, to the intermediate mass stars, which go directly to the red giant branch and then describe blue loops in the HR diagram. At zero rotation, the

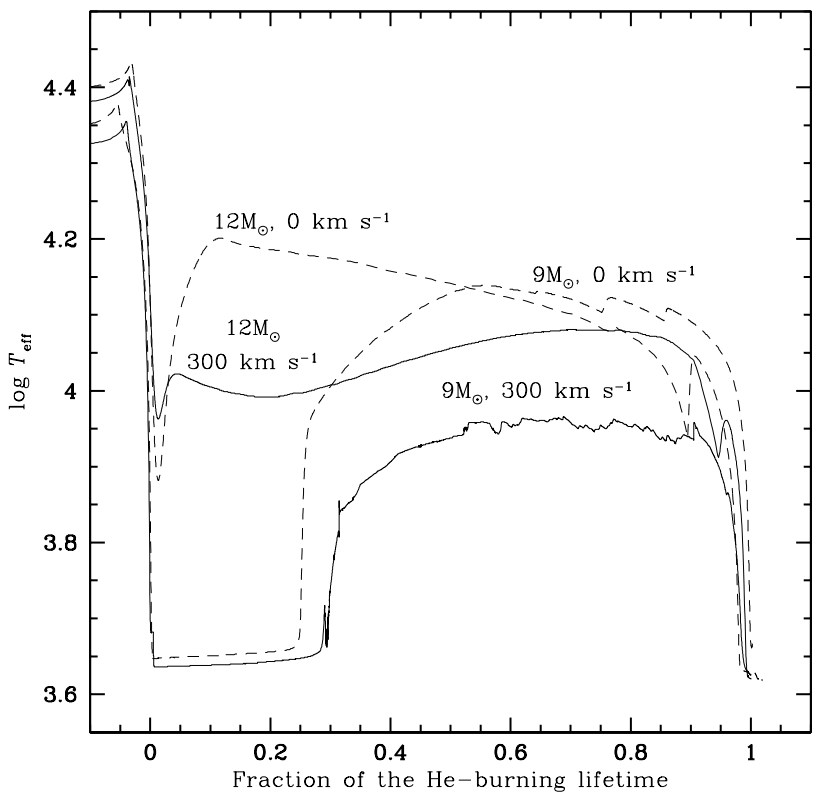

Fig. 11. Evolution of the $T_{\text {eff }}$ as a function of the fraction of the lifetime spent in the He-burning phase for 9 and $12 M_{\odot}$ stars at $Z=0.004$ with $v_{\text {ini }}=0$ and $300 \mathrm{~km} \mathrm{~s}^{-1}$.

$15 M_{\odot}$ model has the "massive star" behaviour and the $9 M_{\odot}$ model shows a most pronounced "blue loop". For $v_{\text {ini }}=0 \mathrm{~km} \mathrm{~s}^{-1}$, the $12 M_{\odot}$ model is just in the transition between the behaviours of nearby models of 9 and $15 M_{\odot}$. The rotating model at $15 M_{\odot}$ is first blue and then goes to the red, while the rotating $9 M_{\odot}$ model goes first to the red, then back to the blue and red again. The behaviour of the rotating $12 M_{\odot}$ is also intermediate between these two, with the consequence that it always stays more or less in the blue, which is surprising at first sight, but well consistent with the mentioned intermediate behaviour. As seen in Sect. 5, this transition zone with almost entirely blue models extends from about 10.5 to $12.2 M_{\odot}$.

The rotating $9 M_{\odot}$ model has a blue loop smaller than for zero rotation; it only extends to the A-type rather than to the B-type range. At a given $L$ and $T_{\text {eff }}$, the average density in a rotating model is much smaller than in the non-rotating one, so that the period will be longer. The result is such that the application of the standard period-luminosity relation will lead for a given observed period to a too high luminosity, if the star was a fast rotator on the MS. A more complete study of the effects of rotation on Cepheids will be made in a further work.

Table 2 shows the $\mathrm{B} / \mathrm{R}$ ratios for the various relevant masses for the models with zero-rotation and $v_{\text {ini }}=$ $300 \mathrm{~km} \mathrm{~s}^{-1}$. Apart from the transition model of $12 M_{\odot}$, which stays almost entirely in the blue as discussed above, we notice that the $B / R$ ratios decrease very much with rotation, being in the range 0.1 to 0.4 for $v_{\text {ini }}=300 \mathrm{~km} \mathrm{~s}^{-1}$. As noted for the $20 M_{\odot}$ model, an average velocity of about $200 \mathrm{~km} \mathrm{~s}^{-1}$ corresponds to a $\mathrm{B} / \mathrm{R}$ ratio of 0.6 . The order of magnitude obtained is satisfactory, however, future comparisons in clusters will need detailed convolution over the IMF and the distribution of rotational velocities 
Table 2. Values of the B/R ratios for models with zero rotation and for models with $v_{\text {ini }}=300 \mathrm{~km} \mathrm{~s}^{-1}$. B means strictly Btype supergiants and $\mathrm{R}$ means $\mathrm{K}$-and $\mathrm{M}$-type supergiants (see text).

\begin{tabular}{ccc}
\hline$M_{\text {ini }}$ & $\mathrm{B} / \mathrm{R}$ & $\mathrm{B} / \mathrm{R}$ \\
& $v_{\text {ini }}=0$ & $v_{\text {ini }}=300$ \\
\hline 25 & 63 & 0.30 \\
20 & 47 & 0.43 \\
15 & 5.0 & 0.24 \\
& & \\
12 & 20.6 & 85 \\
9 & 2.7 & 0.10 \\
\hline
\end{tabular}

in clusters studied at various metallicities. This is beyond the scope of this paper and we now examine the effects in the internal physics which determine the $B / R$ ratio.

\subsection{Stellar physics and the $B / R$ ratio}

There are several studies on the blue-red motions of stars in the HR diagram, for example by Lauterborn et al. (1971), Stothers \& Chin (1979), Maeder (1981), Maeder \& Meynet (1989) and recently by Sugimoto \& Fujimoto (2000). Sugimoto and Fujimoto identify several parameters at the base of the envelope $W, \Theta, V$ and $N$, which play a role in the redwards evolution. Apart from $N$, which is the polytropic index, we may note that the other parameters are all some function of the local gravitational potential. $V$ is the ratio of the gravitational potential to the thermal energy as in Schwarzschild's textbook (1958). The parameter $W$ is given by $W=V / U$, where $U$ is the ratio of the local density to the average internal density. The parameter $\Theta$ is given by $\Theta=\ln \left(\frac{P}{\rho}\right)_{\mathrm{c}}-\ln \left(\frac{P}{\rho}\right)_{\mathrm{env}}$, where the index "c" refers to the center and "env" to the base of the envelope. We can easily check that $\Theta$ is also related to the potential at the center and at the base of the envelope, as well as to the local polytropic index.

We may thus wonder whether most of the effects determining blue vs. red motions in the HR diagram cannot be understood, at least qualitatively, in terms of mainly the gravitational potential of the core. It is very desirable to try to establish some relatively simple scheme for understanding the results of numerical computations. The role of the core gravitational potential for the inflation or deflation of the stellar radius has been emphasized by Lauterborn et al. (1971) in the case of the occurrence of blue-loops for intermediate mass stars (see also Maeder \& Meynet 1989). We shall examine here whether we may extend the very useful "rules" derived by Lauterborn et al. (1971) to the case of massive stars in rotation as studied here. We call $\Phi_{\mathrm{c}}$ the potential of the He-core, which due to a mass-radius relation for the core behaves as $\Phi_{\mathrm{c}} \sim M_{\mathrm{c}}^{0.4}$, where $M_{\mathrm{c}}$ is the core mass. The blue-red motions in the HR diagram mainly depend on the comparison of $\Phi_{c}$ with some critical potential $\Phi_{\text {crit }}(M)$, which grows with the stellar mass. One has

$$
\begin{array}{ll}
\Phi_{\mathrm{c}}>\Phi_{\text {crit }}(M) & \text { Hayashi line } \\
\Phi_{\mathrm{c}}<\Phi_{\text {crit }}(M) & \text { blue location. }
\end{array}
$$

Equations (8) and (9) essentially apply to a steep hydrogen-profile around the core. If this profile is mild, one should account for additional terms (Lauterborn et al. 1971). The main effect can be represented by a parameter $h$ increasing with the amount $\Delta M_{\mathrm{He}}$ of helium in the transition region above the core (formally, a simplified expression for $h$ in the cases considered by Lauterborn et al. (1971) is given by $\log h=8.5 X_{\mathrm{d}} M_{\mathrm{d}} / M$, where $X_{\mathrm{d}}$ is the amplitude of the change of the $\mathrm{H}$-mass fraction over the smooth transition zone, $M_{\mathrm{d}}$ is the width in mass of the transition zone and $M$ the total stellar mass). The value of $h$ also depends on the distribution of this amount of helium $\Delta M_{\mathrm{He}}, h$ is larger if the amount of helium is close to the shell $\mathrm{H}$-burning source. However, if a sufficient amount of helium is brought far from the shell source, in the outer envelope, $h$ may even decrease as suggested by Lauterborn et al. (1971). This also has some consequences, as discussed below. With these remarks, the two above equations become for mild $\mathrm{H}$-profiles
$h \Phi_{\mathrm{c}}>\Phi_{\text {crit }}(M)$
Hayashi line
$h \Phi_{\mathrm{c}}<\Phi_{\text {crit }}(M)$
blue location.

We shall now try to see whether we may describe correctly with relations (10) and (11) the different physical effects influencing the blue-red motions of massive stars:

Mass loss: Mass loss decreases the total stellar mass and thus $\Phi_{\text {crit }}(M)$, which favours a motion towards the Hayashi line. $\Phi_{\mathrm{c}}$ is not very much changed, since the size of the final $\mathrm{He}-$ core is not very different. However, there is more helium near the $\mathrm{H}$-burning shell, which increases the parameter $h$ and also favours the formation of red supergiants.

This description is fully consistent with the well known fact that, due to mass loss, the intermediate convective zone is much less important (cf. Stothers \& Chin 1979; Maeder 1981). A convective zone imposes a polytropic in$\operatorname{dex} N \simeq 1.5$, which implies only a weak density gradient, making the stellar radius smaller and thus keeping the star in the blue. Thus, the physical connexion we have with the interpretation in terms of $\Phi_{\mathrm{c}}$ is the following one. The larger He-burning core with respect to the actual stellar mass together with the higher $\mathrm{He}-$ content in the $\mathrm{H}$-shell region (higher $h$ ) lead to a less efficient $\mathrm{H}$-burning shell, thus there is no large intermediate convective zone and this absence permits a red location of the star in the HR diagram.

Overshooting: The overshooting does not change $\Phi_{\text {crit }}(M)$, but $\Phi_{\mathrm{c}}$ is increased, with no major change of the $\mathrm{H}$-profile and thus of $h$. Clearly, overshooting is thus favouring a redwards motion to the Hayashi line, with 


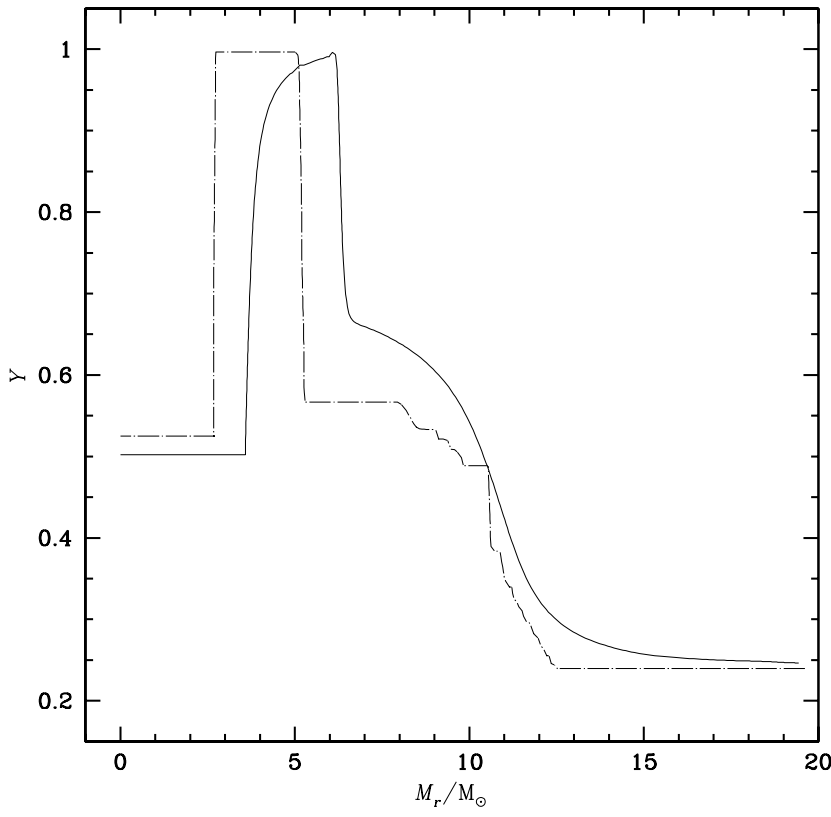

Fig. 12. Comparison of the internal distribution of helium in two models of $20 M_{\odot}$ at the middle of the He-burning phase. The dashed-dot line concerns the models with zero rotation and the continuous line represents the case with $v_{\text {ini }}=300 \mathrm{kms}^{-1}$.

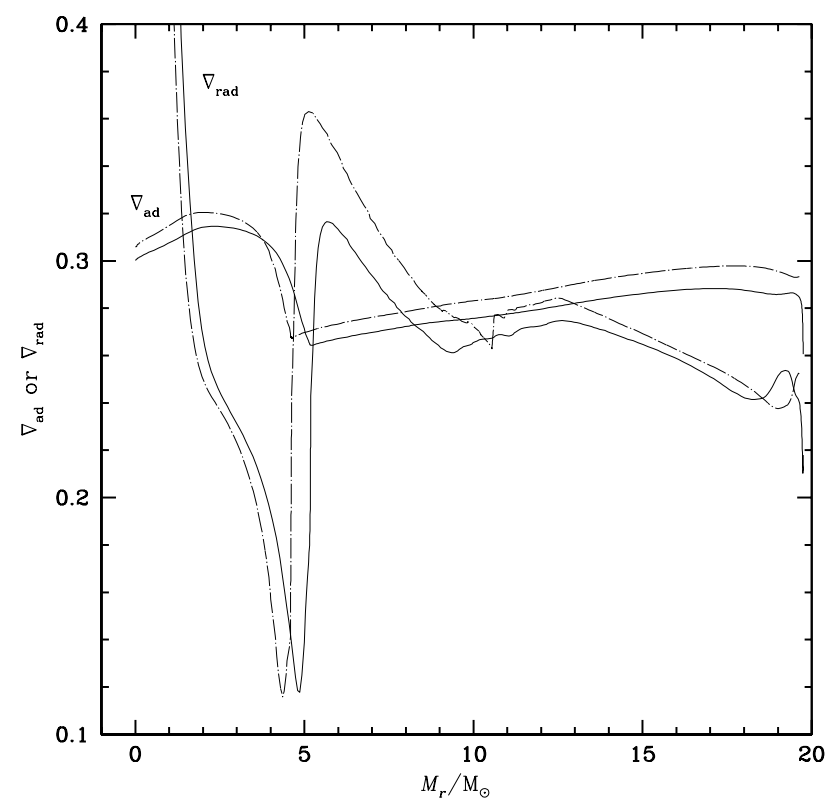

Fig. 13. Comparison of the internal values of $\nabla_{\text {ad }}$ and of $\nabla_{\text {rad }}$ in models of $20 M_{\odot}$ at the very beginning of the He-burning phase $\left(Y_{\mathrm{c}}=0.993\right)$. The dashed-dot lines concern the models with zero rotation and the continuous lines represent the case with $v_{\text {ini }}=300 \mathrm{kms}^{-1}$.

the formation of red supergiants. As for mass loss, the larger core contributes to reduce the intermediate convective zone, which leads to the formation of red supergiants.

Lower metallicity $Z$ : A lower $Z$ decreases the mean molecular weight (essentially because the $\mathrm{He}-$ content is also lower, see Sect. 2.3). This decreases the internal tem-

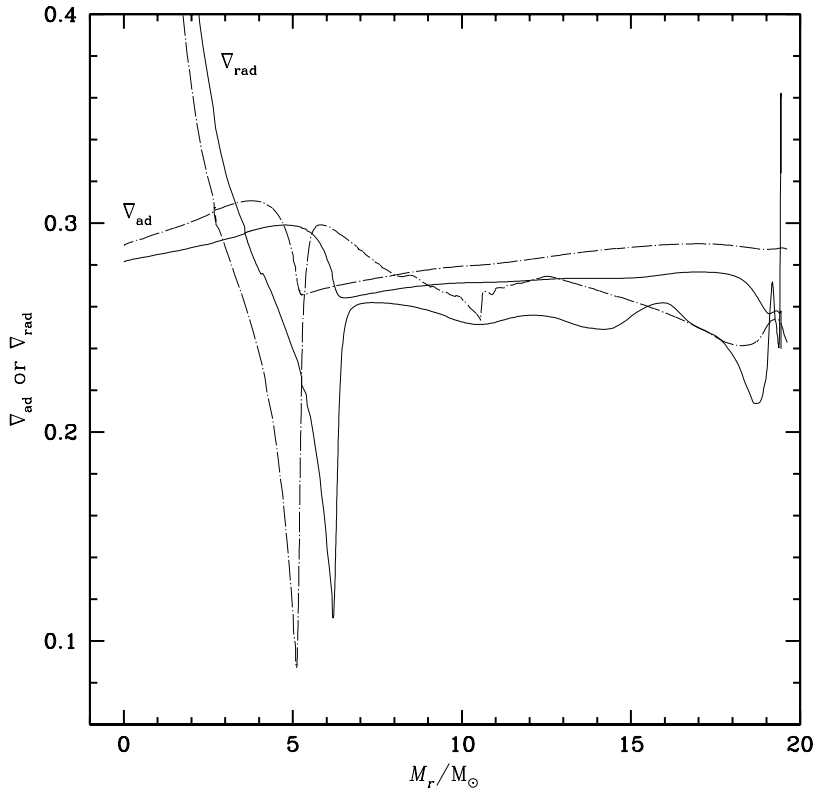

Fig. 14. Comparison of the internal values of $\nabla_{\mathrm{ad}}$ and of $\nabla_{\mathrm{rad}}$ in models of $20 M_{\odot}$ at the middle of the He-burning phase (same model as in Fig. 12). Same remarks as in previous figure.

perature and the luminosity during the MS phase, leading to slightly smaller convective cores, as shown by numerical models (cf. Meynet et al. 1994). This produces smaller $\Phi_{\mathrm{c}}$ which favours a blue location, as is observed.

We also note that a lower $Z$ means a slightly higher electron scattering opacity (due to the higher $\mathrm{H}$-content), which would favour larger cores, however this effect appears as a minor one in the models.

Rotation: The effects of rotation are numerous and subtle, and the balance between them is delicate. We notice the following effects:

1. A first simple effect is that rotation enhances the mass loss rates as described by Eq. (5) and this contributes to favour the formation of red supergiants. However, this effect although significant does not seem to be the dominant one;

2. The mixing in the MS phase leads to a slight extension of the core, which favours a redwards motion during the He-burning phase. This is just like overshooting, thus it may be very difficult, except perhaps by asteroseismological studies of stars with different rotations, to distinguish between the core extension by overshooting or by rotation. An example of this effect can be seen in Fig. 12, which shows models of a $20 M_{\odot}$ star in the middle of the He-burning phase. The core in the non rotating case is $2.7 M_{\odot}$, while it is $3.6 M_{\odot}$ in the rotating case, i.e. $1 / 3$ larger in mass. Although this increase of the core is very significant (in particular for nucleosynthesis), its effect on the blue-red motions appears less important than the effect we now discuss;

3. A mild mixing just outside the core as produced by rotation during the MS phase clearly increases the amount of helium near and above the $\mathrm{H}-$ shell. This is well seen in Fig. 12, (in the two models shown, the 
$\mathrm{H}$-shell is just on the right side of the big He-peak). According to the definition of $h$ given above, this results in a larger value of $h$, which leads Eq. (10) to be satisfied and this favours a red location in the HR diagram. The analysis of the sequence of the models before that illustrated in Fig. 12 shows that the different $\mathrm{H}$-profile of the rotating star is essentially the consequence of the rotational mixing during the MS phase. Thus, the rotational mixing during the MS is the key effect for the formation of red supergiants at low $Z$.

It is satisfactory to see that rules expressed by Eqs. (8) to (11) are fully consistent with what we can deduce by studying the consequences of a change of mean molecular weight. The larger $\mathrm{He}^{-}$core in the rotating models means that a larger fraction of the total luminosity is made in the core ( 0.42 instead of 0.31 for the models of Fig. 12). This means that the $\mathrm{H}$-burning shell in the rotating model produces a smaller fraction of the total luminosity and this contributes to reduce the importance of the convective zone above the $\mathrm{H}-$ burning shell. Simultaneously, the higher He-content near the shell in the rotating case leads to a decrease in the opacity, and this also contributes to reduce the importance of the convective zone associated with the H-burning shell. Figures 13 and 14 show the internal values of $\nabla_{\mathrm{ad}}$ and $\nabla_{\mathrm{rad}}$ in $20 M_{\odot}$ stars at the beginning of the He-burning phase and at the middle of this phase respectively. We notice the smaller convective zone associated with the $\mathrm{H}$-burning shell in the rotating model in Fig. 13 and its earlier disappearance in Fig. 14. As long as it exists, the convective zone maintains a small polytropic index over the concerned region, and the larger this region is, the smaller the radius. Only when the intermediate convective zone disappears, the star reaches the red supergiant location in the HR diagram. We see that the earlier disappearance of this intermediate convective zone in rotating stars favours their earlier evolution towards red supergiants, consistent with the result of Eqs. (10) and (11);

4. We may wonder what are the respective effects of mixing on the MS and during the He-burning phase. What we have shown above is just the consequence of the mixing during the MS phase. Some tests have been made and show the following. If we arbitrarily stop the mixing during the He-burning phase, this makes little difference. However, if on the contrary we arbitrarily enhance the mixing during the He-burning phase, this maintains the star in a blue location in the HR diagram. This result is in agreement with the effect mentioned above and discussed by Lauterborn et al. (1971). The parameter $h$ normally grows with $\Delta M_{\mathrm{He}}$, however it may reach a maximum and then decrease, if helium is brought far enough from the $\mathrm{H}_{-}$ shell. Thus, strong mixing in the He-burning phase by spreading helium throughout the star may make $h$ decrease, which according to Eq. (11) leads to a blue location in the HR diagram.
Why does this occur physically? Mixing of helium also implies mixing of hydrogen. For moderate mixing, the $\mathrm{H}-$ burning shell becomes more active, due to the increase of its $\mathrm{H}-$ content and $T$. This activates the intermediate convective zone associated with the $\mathrm{H}$-shell. Additional He at the surface also decreases the opacity and favours bluer stars. For more extreme cases of mixing both in the MS and subsequent phases, one would move to the case of almost homogeneous stars, which occurs for fast rotators such as the $60 M_{\odot}$ model at $Z=0.02$ with a $v_{\text {ini }}$ of $\sim 400 \mathrm{~km} \mathrm{~s}^{-1}$ (cf. Meynet \& Maeder 2000). In this case, a bluewards evolution in the HR diagram occurs during the MS phase, as shown by Schwarzschild (1958).

\section{Evolution of the chemical abundances at the surface}

As already emphasized in previous works (see Maeder \& Meynet 2000a and references therein; Meynet \& Maeder 2000; Heger \& Langer 2000), rotation significantly modifies the evolution of the surface abundances. Figure 15 illustrates the changes of the nitrogen to carbon ratios $\mathrm{N} / \mathrm{C}$ from the ZAMS to the red supergiant stage for some $20 M_{\odot}$ stellar models. For non-rotating stars, the surface enrichment in nitrogen only occurs when the star reaches the red supergiant phase; there, CNO elements are dredged-up by deep convection.

For rotating stars, $\mathrm{N}$-excesses already occur during the MS phase. These $\mathrm{N}-$ excesses are not necessarily accompanied by surface He-enrichments. From Table 1, one sees that for the rotating models, well before any change of the surface $\mathrm{He}-$ abundance occurs, the $\mathrm{N} / \mathrm{C}$ and $\mathrm{N} / \mathrm{O}$ ratios undergo significant changes. This results from the effects of transport on the very strong gradients of the CNO elements which rapidly build up inside the star, (we note that mass loss at very high rates would do the same). Thus the existence of $\mathrm{N}$-rich stars with a normal He abundance is easily explained.

After the MS phase, the $\mathrm{N} / \mathrm{C}$ ratios at the surface of rotating stars continue to increase during the red supergiant phase. At low metallicity, some increase also occurs when the star is crossing the HR diagram (see below). The $\mathrm{N} / \mathrm{C}$ ratios obtained at the end of the $\mathrm{He}$-burning phase are much higher in rotating models than in non-rotating ones.

From Fig. 15, one sees that at a given metallicity, the higher the initial rotational velocity, the more important are the surface enrichments at the end of the MS phase. This results from the stronger angular velocity gradients and transport in faster rotating stars. The increase from $v_{\text {ini }}=200$ to $300 \mathrm{~km} \mathrm{~s}^{-1}$ produces greater relative changes than the increase from 300 to $400 \mathrm{~km} \mathrm{~s}^{-1}$ (see Fig. 15). Likely, this saturation effect results from the compensating action of two effects. On the one hand, when the rotational velocity increases, the transport of the chemical species by the shear becomes more efficient. On the other hand, when evolution proceeds, this more efficient mixing produces smoother chemical and angular momentum 


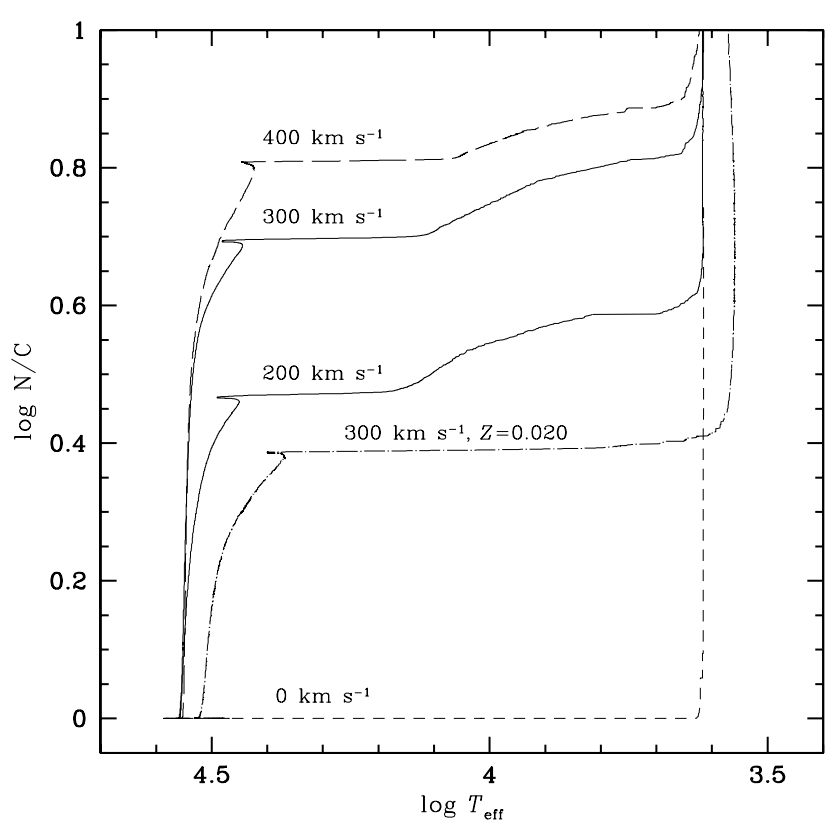

Fig. 15. Evolution as a function of $\log T_{\text {eff }}$ of the abundance ratio $\mathrm{N} / \mathrm{C}$ where $\mathrm{N}$ and $\mathrm{C}$ are the surface abundances of nitrogen and carbon respectively. The abundance ratios are normalized to their initial value. The tracks are for $20 M_{\odot}$ at $Z=0.004$ with different initial rotational velocities. The dot-long dashed line shows the evolution of a rotating $20 M_{\odot}$ at solar metallicity $\left(v_{\text {ini }}=300 \mathrm{kms}^{-1}\right)$.

gradients and thus results in a slowing down of the transport processes. From Table 1, one sees that, in general, the $\mathrm{N}$ surface enrichments at the end of the MS phase are higher for higher initial masses. As explained by Maeder (1998) this results from the fact that, when the initial mass increases, the ratio of the mixing timescale for the chemical elements to the MS lifetime decreases.

\subsection{Surface enrichments at $Z=0.004$ and 0.020}

Very interestingly, for a given value of the initial velocity, the lower the initial metallicity, the greater the surface $\mathrm{N}$-enrichments. The mixing of the chemical elements is more efficient at low metallicity essentially because the metal poor stars are more compact and have greater angular velocity gradients. Thus the mixing timescales for the chemical elements, which is about $D_{\text {shear }} / R^{2}$, are smaller at lower metallicities.

As already briefly mentioned above, the models at low metallicity present enhancements of their $\mathrm{N} / \mathrm{C}$ ratios during their crossing of the HR diagram, while the solar models do not present any enrichment during this phase. This is a consequence of the fact that at low metallicity, the stars begin to burn helium in their core in the blue part of the HR diagram. This slows down the evolution towards the red and thus gives more time for the transport processes to bring $\mathrm{CNO}$ processed material at the surface, while the star is still in the blue.

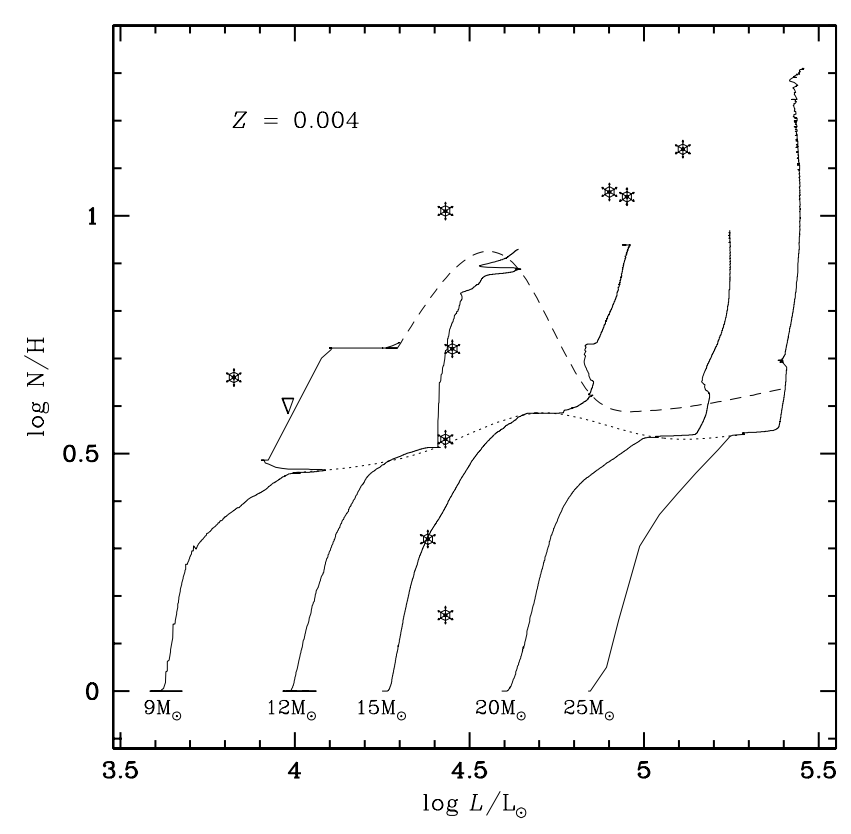

Fig. 16. Surface nitrogen to hydrogen ratios as a function of luminosity. The $\mathrm{N} / \mathrm{H}$ ratios are normalized to their initial value. The continuous lines represent evolutionary tracks for rotating models with $v_{\text {ini }}=300 \mathrm{~km} \mathrm{~s}^{-1}$. The initial masses are indicated. The dotted line indicates the $\mathrm{N} / \mathrm{H}$ ratios obtained at the end of the MS phase. The dashed line shows this same relation when $\log T_{\text {eff }}$ is equal to 3.9 . For the $9 M_{\odot}$ model which presents a blue loop, the value corresponds to the last crossing at $\log T_{\text {eff }}=3.9$ before the star becomes a red supergiant. The points represent the observed values from Venn (1999), an inverted triangle is plotted when only an upper limit is given.

\subsection{Comparisons with the observations}

Figure 16 shows the evolution of $\mathrm{N} / \mathrm{H}$, the nitrogen to hydrogen ratios, at the surface of our rotating models with $v_{\text {ini }}=300 \mathrm{~km} \mathrm{~s}^{-1}$. The ratios are normalized to their initial value. The evolutions of the $\mathrm{N} / \mathrm{H}$ ratio at the surfaces of the non-rotating and rotating stars are qualitatively similar to the behaviours described above for the $\mathrm{N} / \mathrm{C}$ ratio. Let us simply say here that in the plane of Fig. 16, the non-rotating models predict that stars are either on the horizontal line defined by $\log \mathrm{N} / \mathrm{C}=0$ or along the line $\log \mathrm{N} / \mathrm{C}=0.5-0.6$, which corresponds to the position of the non-rotating stars having undergone the first dredgeup. No stars are predicted outside these two regions.

Obviously, the observed values obtained by Venn (1999) for A and F supergiants in the SMC are not concentrated along the horizontal lines predicted by the nonrotating models. Two observed supergiants show nitrogen enhancements well below the values predicted by the first dredge-up in non-rotating models. Therefore these cannot be on a blue loop. Instead, they are probably on their way from the MS to the red giant branch and have undergone some mixing in the early stage of their evolution. A- and F-type supergiants observed by Venn (1999) are also observed well above the line corresponding to the first dredge- up of non-rotating models. Again, this is an indication of an extra mixing process active in massive stars. 
Previous studies have shown that for galactic blue supergiants, rotation appears as a very promising process to account for the observed surface enrichments (see Heger \& Langer 2000; Paper V; Meynet 2001). Can we say the same at low metallicity? In Fig. 16, the dotted and the dashed lines define the evolution between the end of the MS phase and the stage corresponding to $\log T_{\text {eff }}=3.9$. For stars more massive than about $15 M_{\odot}$, the $\mathrm{N}$-excesses do not change very much during this phase. For the stars that present a blue loop episode (even a "partial blue loop" episode like the $12 M_{\odot}$ model), important enrichments occur before the star settles into the red supergiant stage.

If all the stars were rotating with an initial velocity of $300 \mathrm{~km} \mathrm{~s}^{-1}$, these models would predict that the observed A-type supergiants would be around the dashed line in Fig. 16. For lower initial velocities the $\mathrm{N}-$-enrichments can be everywhere between the horizontal line " $\log \mathrm{N} / \mathrm{C}=0$ " and the dashed line. For higher initial rotational velocities, the $\mathrm{N}$-enrichments would be higher than the dashed line. Some very $\mathrm{N}$-rich stars at high luminosities (such as the N-rich stars with $\mathrm{N} / \mathrm{H}>10$ and $\left.\log L / L_{\odot}>\sim 4.2\right)$ could be accounted for either by stars with very high initial rotation now on their redward tracks, or by very rapid rotators which, after a red supergiant phase, are going back to the blue. Thus we can draw the same conclusions as the ones deduced at solar metallicity, namely that there is observational evidences for an extra mixing process active in massive stars, and that rotational mixing appears as a very likely process to drive the extra mixing necessary to account for the observations.

Moreover, the higher enrichments obtained at metallicity lower than solar is in agreement with the observations by Venn $(1995,1999)$. Comparing the range of the $\mathrm{N} / \mathrm{H}$ values measured at the surface of A-type supergiants in the Galaxy and in the SMC, she obtained that in the $\mathrm{SMC}$, the $\mathrm{N} / \mathrm{H}$ values cover an interval three times greater than in the Galaxy. At $\log T_{\text {eff }}=3.9$ the $\mathrm{N} / \mathrm{H}$ ratio at the surface of the $20 M_{\odot}$ model at $Z=0.004$ with $v_{\text {ini }}=300 \mathrm{~km} \mathrm{~s}^{-1}$ is two times greater than the ratio at the surface of the similar model at solar metallicity. For the $\mathrm{N} / \mathrm{C}$ ratio the enhancement amounts to a factor 2.5 , as can be seen from Fig. 15. Thus, even if the distribution of the rotational velocities are the same at both metallicities, one expects that the low metallicity stars are relatively richer in nitrogen. If there are initially more fast rotating stars at low metallicity, this will reinforce this trend.

Thus rotation not only can account for the observations of N-rich blue supergiants at both solar and SMC metallicity, but it might also account for the fact that at low metallicity the maximum enrichments observed are greater than at solar metallicity. Finally, we point out that in the present models we have found no primary nitrogen produced. This is not a difficulty, because the evidence for the existence of some primary nitrogen concern metallicities still lower than that of the SMC (cf. Henry et al. 2000). An interesting question for future models is to see whether massive star models with rotation at much lower $Z$ produce any primary nitrogen.

\section{Conclusions}

There are 3 main conclusions of this work:

1. The ratios $\Omega / \Omega_{\text {crit }}$ of the angular velocity to the breakup angular velocity grow quite a lot during the evolution of massive stars at low $Z$, contrary to the case of massive stars at solar metallicity. This implies that at lower $Z$ a larger fraction of massive stars is close to the break-up velocity. This effect results from the lower mass loss rates and from the significant outwards transport of angular momentum by meridional circulation. This is very consistent with the observational results by Maeder et al. (1999), who observed a larger fraction of Be stars, when passing from Galaxy, to LMC and SMC. The question arises whether the initial distributions of the stellar rotation in galaxies of different $Z$ are different. Some arguments in the quoted paper suggest that it might be the case, but this is still very uncertain. However, we show here that even if the distribution of $v \sin i$ is not different for massive stars with lower $Z$, the net result of the evolution on the MS leads to much faster rotational velocities at the end of the MS phase, when the metallicity is lower;

2. Due mainly to the additional helium brought near the $\mathrm{H}$-burning shell by rotational mixing and the larger He-core, which both lead to a less efficient $\mathrm{H}$-burning shell and a smaller associated convective zone, the stellar radius of rotating stars is permitted to inflate during the He-burning phase. These models account for the formation of numerous red supergiants at low $Z$, with a blue to red supergiant ratio $\mathrm{B} / \mathrm{R}$ consistent with the observations in the SMC cluster NGC 330;

3. While the standard models with mass loss usually predict no N/C enrichments on the main sequence and in the $\mathrm{B}$ - to $\mathrm{F}$-type supergiants, unless they have experienced convective dredge-up in red supergiants, the models with rotation predict progressive $\mathrm{N} / \mathrm{C}$ and $\mathrm{N} / \mathrm{O}$ enrichments along the evolutionary tracks. The predicted $\mathrm{N}$ excesses are in general larger at larger stellar luminosities. Also, higher relative excesses of $\mathrm{N}$ are predicted at lower metallicity by the present models. The comparisons with the observations by Venn $(1998,1999)$ of A-type supergiants show a good agreement for the size of the $\mathrm{N}$-enrichments, as well as for the fact that they are larger at lower $Z$.

The major interrogation for the future lies in the line of the first conclusion. Is rotation more and more important as we go to massive stars of lower $Z$ ? This is a question for which great observational efforts need to be made. If the answer is yes, for whatever reason (star formation or effect of MS evolution), this will imply that rotation is a dominant effect and has important consequences for the interpretation of the spectra of high redshift galaxies and for the early nucleosynthesis. 


\section{References}

Angulo, C., Arnould, M., Rayet, M., et al. 1999, Nucl. Phys. A, 656,3

Arnett, D. 1991, ApJ, 383, 295

Bertelli, G., Bressan, A., \& Chiosi, C. 1985, A\&A, 150, 33

Brocato, E., \& Castellani, V. 1993, ApJ, 410, 99

Charbonnel, C., Meynet, G., Maeder, A., Schaller, G., \& Schaerer, D. 1993, A\&AS, 101, 415

Chin, C. W., \& Stothers, R. B. 1990, ApJS, 73, 821

Claret, A., \& Gimenez, A. 1998, A\&AS, 133, 123

Caughlan, G. R., \& Fowler, W. A. 1988, Atomic Data and Nuclear Data Tables, 40, 283

Caughlan, G. R., Fowler, W. A., Harris, M. J., \& Zimmerman, B. A. 1985, Atomic Data and Nuclear Data Tables, 32, 197

de Jager, C., Nieuwenhuijzen, H., \& van der Hucht, K. A. 1988, A\&AS, 72, 259

Fagotto, F., Bressan, A., Bertelli, G., \& Chiosi, C. 1994, A\&AS, 104,365

Fernandes J., Lebreton Y., Baglin, A., \& Morel, P. 1998, A\&A, 338,455

Fliegner, J., \& Langer, N. 1995, Models of rotating Wolf-Rayet stars, in ed. K. A. van der Hucht, \& P. M. Williams, IAU Symp. 163, Wolf-Rayet Stars: Binaries, Colliding Winds, Evolution (Kluwer), 326

Flower, P. J. 1996, ApJ, 469, 355

Friend, D. B., \& Abbott, D. C. 1986, ApJ, 311, 701

Girardi, L., Bressan, A., Bertelli, G., \& Chiosi, C. 2000, A\&AS, 141,371

Heger, A., \& Langer, N. 1998, A\&A, 334, 210

Heger, A., \& Langer, N. 2000, ApJ, 544, 1016

Heger, A., Woosley, S. E., \& Langer, N. 2000, ApJ, 528, 368

Henry, R. B. C., Edmunds, M. G., \& Köppen, J. 2000, ApJ, 541,660

Herrero, A., Puls, J., \& Villamariz, M. R. 2000, A\&A, 354, 193

Humphreys, R. M. 1979. ApJ, 231, 384

Humphreys, R. M., \& McElroy, D. B. 1984, ApJ, 284, 565

Iglesias, C. A., \& Rogers, F. J. 1996, ApJ, 464, 943

Izotov, Y. I., Thuan, T. X., \& Lipovetsky, V. A. 1997, ApJS, 108,1

Kippenhahn, R., \& Weigert, A. 1990, Stellar Structure and Evolution (Springer Verlag), 468

Kucinskas, A., Vansevicius, V., Sauvage, M., \& Tanabe, T. 2000, A\&A, 353, L21

Kudritzki, R. P., \& Puls, J. 2000, ARA\&A, 38, 613

Lamers, H. J. G. L. M., Snow, T. P., \& Lindholm, D. M. 1995, ApJ, 455, 269

Langer, N. 1997, The Eddington Limit in Rotating Massive Stars, ed. A. Nota, \& H. Lamers, Luminous Blue Variables: Massive Stars in Transition, ASP Conf. Ser., 120, 83

Langer, N. 1998, A\&A, 329, 551

Langer, N., \& Maeder, A. 1995, A\&A, 295, 685
Lauterborn, D., Refsdal, S., \& Weigert, A. 1971, A\&A, 10, 97 Maeder, A. 1981, A\&A, 102, 401

Maeder, A. 1987, A\&A, 178, 159

Maeder, A. 1997, A\&A, 321, 134 (Paper II)

Maeder, A. 1998, The Physics of Rotational Mixing in Massive Stars, ed. I. Howarth, Boulder-Munich II: Properties of Hot, Luminous Stars, ASP Conf. Ser., 131, 85

Maeder, A., \& Mermilliod, J. C. 1981, A\&A, 93, 136

Maeder, A., \& Meynet, G. 1989, A\&A, 210, 155

Maeder, A., \& Meynet, G. 2000a, ARA\&A, 38, 143

Maeder, A., \& Meynet, G. 2000b, A\&A, 361, 159 (Paper VI)

Maeder, A., \& Zahn, J. P. 1998, A\&A, 334, 1000 (Paper III)

Maeder, A., Grebel, E., \& Mermilliod, J. C. 1999, A\&A, 346, 459

Meylan, G., \& Maeder, A. 1982, A\&A, 108, 148

Meynet, G. 2000, Evolution of rotating massive stars into the Wolf-Rayet phase at solar metallicity, ed. A. Lançon, Massive Stellar Clusters, ASP Conf. Ser., 105

Meynet, G. 2001, in Mixing and Diffusion in Stars: Theoretical Predictions Observational Constraints, ASP Conf. Ser., ed. D. VandenBerg, \& J. P. Zahn, in press

Meynet, G., \& Maeder, A. 1997, A\&A, 321, 465 (Paper I)

Meynet, G., \& Maeder, A. 2000, A\&A, 361, 101 (Paper V)

Meynet, G., Maeder, A., Schaller, G., Schaerer, D., \& Charbonnel, C. 1994, A\&AS, 103, 97

Nugis, T., \& Lamers, H. J. G. L. M. 2000, A\&A, 360, 227

Pagel, B. E. J., Simonson, E. A., Terlevich, R. J., \& Edmunds, M. G. 1992, MNRAS, 255, 325

Peimbert, M., Peimbert, A., \& Ruiz, M. R. 2000, ApJ, 541, 688

Sackmann, I. J., \& Anand, S. P. S. 1970, ApJ, 162, 105

Schaller, G., Schaerer, D., Meynet, G., \& Maeder, A. 1992, A\&AS, 96, 269

Schwarzschild, M. 1958, Structure and Evolution of the Stars (Dover Publ.), 269

Stothers, R. B., \& Chin, C. W. 1979, ApJ, 233, 267

Stothers, R. B., \& Chin, C. W. 1992, ApJ, 390, 136

Sugimoto, D., \& Fujimoto, M. Y. 2000, ApJ, 538, 837

Talon, S., \& Zahn, J. P. 1997, A\&A, 317, 749

Talon, S., Zahn, J. P., Maeder, A., \& Meynet, G. 1997, A\&A, 322,209

Thuan, T. X., \& Izotov, Y. I. 1998, Space Sci. Rev., 84, 83

Vanbeveren, D., de Loore, C., \& van Rensbergen, W. 1998, Astron. Astrophys. Rev., 9, 63

Venn, K. A. 1995, ApJ, 449, 839

Venn, K. A. 1998, in Boulder-Munich II: Properties of Hot, Luminous Stars, ed. I. Howarth, ASP Conf. Ser., 131, 177

Venn, K. A. 1999, ApJ, 518, 405

Willson, L. A. 2000, ARA\&A, 38, 573

Zahn, J. P. 1992, A\&A, 265, 115

Zijlstra, A., Loup, C., Waters, L. B. F. M., et al. 1996, MNRAS, 279,32 